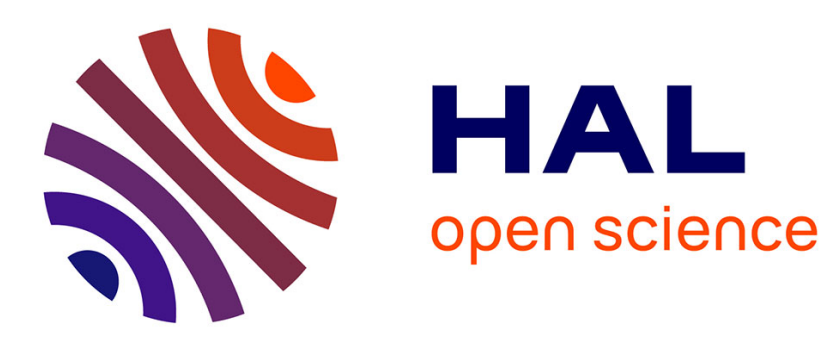

\title{
Interaction between biofilm growth and NAPL remediation: A pore-scale study
}

M. Benioug, Fabrice Golfier, P. Fischer, C. Oltean, M. Buès, X. Yang

\section{To cite this version:}

M. Benioug, Fabrice Golfier, P. Fischer, C. Oltean, M. Buès, et al.. Interaction between biofilm growth and NAPL remediation: A pore-scale study. Advances in Water Resources, 2019, 125, pp.8297. 10.1016/j.advwatres.2019.01.011 . hal-02007310

\section{HAL Id: hal-02007310 https://hal.univ-lorraine.fr/hal-02007310}

Submitted on 13 Feb 2019

HAL is a multi-disciplinary open access archive for the deposit and dissemination of scientific research documents, whether they are published or not. The documents may come from teaching and research institutions in France or abroad, or from public or private research centers.
L'archive ouverte pluridisciplinaire HAL, est destinée au dépôt et à la diffusion de documents scientifiques de niveau recherche, publiés ou non, émanant des établissements d'enseignement et de recherche français ou étrangers, des laboratoires publics ou privés. 


\section{Interaction between Biofilm Growth and NAPL Remediation: A Pore-scale Study}

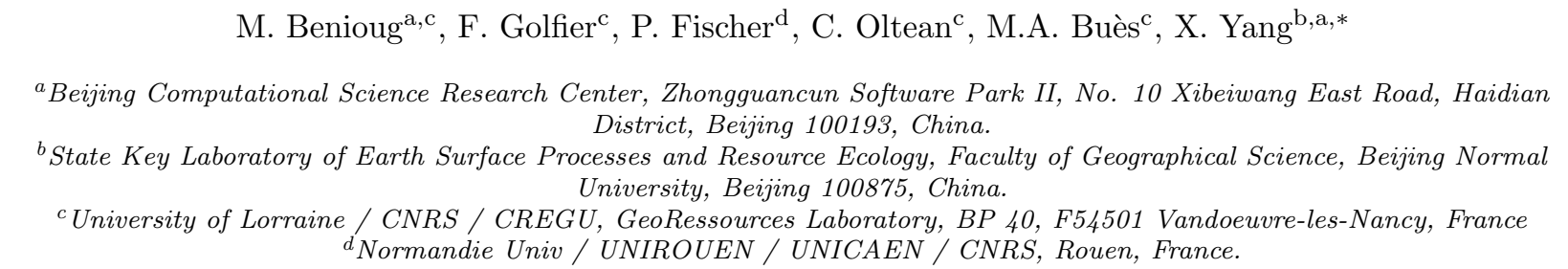
${ }^{b}$ State Key Laboratory of Earth Surface Processes and Resource Ecology, Faculty of Geographical Science, Beijing Normal University, Beijing 100875, China.

\section{Abstract}

In this paper, we introduce a pore-scale model to study the interaction between biofilm growth and nonaqueous-phase-liquid (NAPL) dissolution. Liquid flow and dissolved NAPL transport are coupled with a biofilm growth model to correctly describe the complex dynamics of the processes including fluid flow, NAPL dissolution/biodegradation and biofilm growth. Fluid flow is simulated using an immersed boundary-lattice Boltzmann model; while solute transport is described by a cut-cell finite volume method. A uniform dissolution approach is also adopted to capture the temporal evolution of trapped blobs. Spatio-temporal distribution of the biomass are investigated using a cellular automaton algorithm combined with the immersed boundary method. Simulations focused on the dissolution of NAPL in both abiotic and biotic conditions are conducted to assess the capability of the model. In abiotic conditions, we analyze the effects of the hydrodynamic regimes and the spatial distribution of NAPL blobs on the dissolution rate under different assumptions (blob size and Péclet number). In biotic conditions, a series of scenarios are also investigated (spatial distribution, reaction kinetics and NAPL-induced toxicity). Finally, this model is used to evaluate the pore scale relevance of a local equilibrium assumption between fluid phase and biofilm phase in the vicinity of the NAPL source.

Keywords:

NAPL biodegradation, lattice Botzmann method, immersed boundary method, biofilm growth, porous media, cellular automata

\section{Introduction}

Non-aqueous phase liquids (NAPLs) are important but challenging problems for traditional groundwater remediation (Kent and Bianchi, 2001, Lee et al., 2007). NAPLs are the hydrocarbons that include the halogenated chemical solvents and petroleum products (such as PCB, PAH, BTEX, etc.) and often contaminate

*xfyang@bnu.edu.cn 
the subsurface after an accidental spill or oil tank leakage (Mercer and Cohen, 1990, Miller et al., 1998).

These contaminants remain trapped in the form of immiscible droplets within the aquifers, becoming a persistent source of contamination that is difficult to remediate. Predicting the fate of the contaminants requires characterizing all the mechanisms involved in the coupled processes, especially biodegradation, which can occur in the vicinity of the contaminated source or further, within the dissolved plume (Fig. 1).

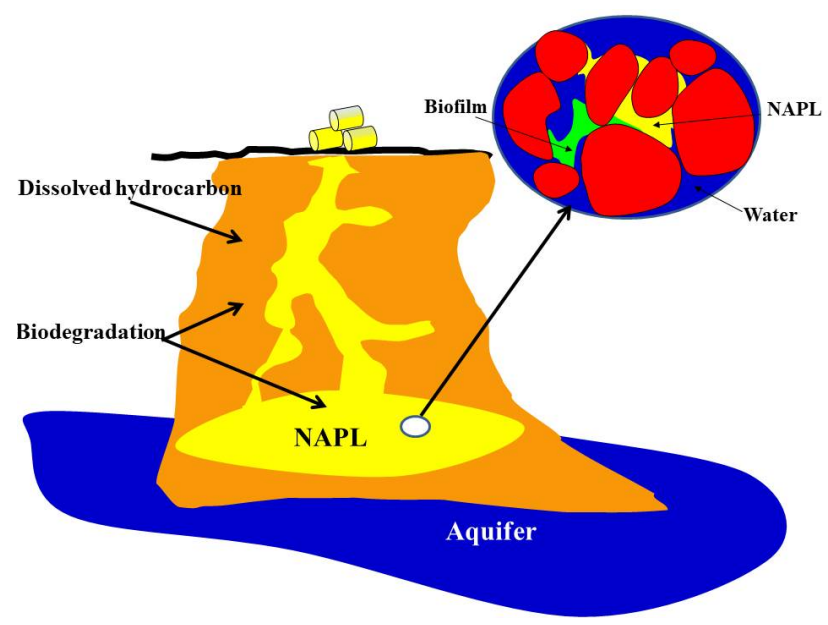

Figure 1: Sketch of an aquifer contaminated by NAPLs.

One of the major difficulties to model such processes and advance the understanding of NAPLs transport in porous media with biofilm (the bacterial phase) is the complex interplay between biological and physicochemical phenomena (Vega et al., 2014, Brackman et al., 2009, Chmielewski and Frank, 2003, Eberl et al., 2001, Krefta et al., 2001, Picioreanu et al., 2000a, Eberl et al., 2000, Mah and O'Toole, 2001, Decho, 2000, Wingender et al., 1999). These processes also have multiscale nature in both time and space (van Loosdrecht et al., 2002, Picioreanu et al., 2000b, Kreft et al., 1998). The study of NAPL biodegradation in porous media is of growing interest due to many environmental applications where biofilm plays an essential role, such as for example, the bioremediation of contaminated sites (Al-Bader et al., 2013, Singh et al., 2006) or the development of bio-barriers for aquifer protection (Huang et al., 2011, Seo et al., 2009).

In recent decades, extensive efforts have been devoted to study contaminant transport in porous media with biofilms. Nevertheless, whether is at the pore scale or macroscopic scale, these studies (Ebigbo et al., 2013, Golfier et al., 2009, Kreft et al., 1998) were focused, in the most part, on the areas away from the source of contamination. Additionally most of the previous work were interested in dissolution of residual NAPL saturation and limited to abiotic conditions (Mateas et al., 2017, Imhoff et al., 1994, Powers et al., 1994, 1992, Miller et al., 1990). Despite the importance of these contributions to understand the mechanisms involved during biotic or abiotic dissolution of NAPLs, it is still challenging to predict the fate of the contaminant without considering the biological activities close to the source of contamination. At present, to our best 
knowledge, no numerical study has modeled the bacterial growth mechanisms at the pore scale, specifically those involving NAPL sources, which are indeed believed to be hot spots of the biological activities.

Many studies (Paulsen et al., 1989, Yang and L. McCarty, 2000, Armstrong and Wildenschild, 2012) have shown that bacterial metabolism of the oil-degrading bacteria leads to the production of biosurfactant products in the presence of NAPLs. These products are active surfactants that reduce the interfacial tension at the surface of the pure NAPL droplet so as to destabilize and break it into small droplets evacuated by the flow. Experiments performed in 2D transparent micromodels by Armstrong and Wildenschild (2012) compared the effectiveness of the abiotic and biotic degradation of NAPLs and the role of each parameter on the macroscopic process. The authors concluded that biodegradation is optimal in the presence of biosurfactant-producing biomass, benefiting simultaneously from the enhanced mobilization and consumption effects of NAPLs. The results of Paulsen et al. (1989) also deserve to be reported as they highlighted the impact of biosurfactant production on the dissolution process of a hydrocarbon phase. Paulsen et al. (1989) experimentally investigated the influence of biosurfactants on a NAPL droplet at the pore scale. The laboratory images showed that the growth of the biofilm with associated biofilaments developed preferentially around and even on the droplet. An attempt to upscale this bio-enhanced NAPL dissolution was proposed by Bahar et al. (2016). Nevertheless, NAPLs also constitute a xenophobic agent for bacteria that may potentially inhibit their growth. In their experiment, Singh and Olson (2010) followed the evolution of the concentration of living and dead bacteria around a droplet of TCE placed in the presence of Pseudomonas sp. Initially, biofilm was developed uniformly at the periphery of the droplet. After 5 minutes, living bacteria migrated gradually away from TCE. At the same time, an increase in the number of dead cells in the vicinity of the droplet was observed. This area of dead cell accumulation kept increasing with time. This experiment highlighted the influence of the toxic effect of NAPLs, which, at high concentrations, caused the death of nearby bacteria. The influence of toxicity caused by the dissolution enhanced through the biodegradation of NAPLs was also studied at macroscopic scale via numerical simulations ( Chu et al., 2004, Gallo and S.M. Hassanizadeh, 2002).

The aim of this paper is to present a numerical model with the capability to predict the biodegradation of the NAPLs in porous medium at the pore scale. First, we conduct numerical simulations to study the dissolution of NAPLs in abiotic conditions under different hypotheses (blob size and Péclet number). Second, we focus on the phenomenon of NAPL dissolution in the presence of bacteria. Different conditions are considered (spatial distribution, reaction kinetics, toxicity effect), and their impact on the dissolution process are assessed. Finally, the current pore-scale model is used to evaluate the relevance of a local equilibrium assumption between the fluid and the biofilm phases close to the NAPL source.. 


\section{Numerical Model}

The numerical model proposed here extends the previous biofilm growth model (Benioug et al., 2017) by introducing an additional NAPL phase. Since in the present study, we investigate the mutual interaction between NAPL phase and bacterial activity, four phases are included in the current model: solid $(s)$, fluid $(f)$, biofilm $(b)$ and NAPL $(h)$. In addition to the assumptions introduced in (Benioug et al., 2017) (monospecies biofilm, nutrient transport only by diffusion in the biofilm phase), we further assume that:

- NAPL is assumed trapped as an immobile phase within the water-wet porous matrix.

- Residual NAPLs are trapped in the form of perfectly circular blobs (in $2 D$ ). In addition, flows passing through the NAPL interface (in the case of a triple interface with the fluid and the biofilm for the same blob) are supposed not to be sufficient to overcome the forces of surface tension (no breakage of the blobs). In other words, the radius of each NAPL blob decreases over time as a function of the sum of the fluxes exchanged with the surrounding medium.

- NAPL is a pure phase composed of a single component which is biodegradable, and acting as the electron donor in the redox process. Other substances (electron acceptor, mineral salts, amino acids) that are necessary for the survival of bacteria are assumed to be in excess.

- Diffusion of water within the NAPL phase is negligible.

- Biofilm may develop at the fluid and NAPL interfaces. Bacteria produce biosurfactants whose concentration is assumed to be constant and are present only within the biofilm phase (no transport of the biosurfactants produced into the fluid phase and no variability of the interfacial conditions as a function of the surfactant concentration).

- Only the increase in solubility of the dissolved hydrocarbon (via the partition coefficient $K_{b h}$ ) in the presence of biosurfactants is considered. The change of the surface tension is not taken into account. Removal of NAPL droplets by water flow is also assumed to be negligible in the dissolution process.

- Biodegradation of the dissolved hydrocarbon within the biofilm phase is governed by the Haldane kinetics to account for the inhibition effects (Saravanan et al., 2008).

\subsection{Fluid Flow}

In the fluid phase $(f)$, we consider steady-state, incompressible and Newtonian flow at low Reynolds number. The mass balance and momentum conservation equations are described by the Stokes equations as follows:

$$
\nabla \cdot \mathbf{u}=0
$$




$$
\rho_{f} \mathbf{u} \cdot \nabla \mathbf{u}=-\nabla p+\mu_{f} \nabla^{2} \mathbf{u}
$$

and no-slip boundary conditions for fluid-solid interfaces $A_{f s}$, fluid-biofilm $A_{f b}$ and fluid-NAPL $A_{f h}$ are employed as:

$$
\begin{array}{lll}
\text { B.C.1 } & \mathbf{u}=0, & \text { at } A_{f b} \\
\text { B.C.2 } & \mathbf{u}=0, & \text { at } A_{f s} \\
\text { B.C.3 } & \mathbf{u}=0, & \text { at } A_{f h}
\end{array}
$$

where $\mathbf{u}, p, \mu_{f}, \rho_{f}$ are respectively the velocity, pressure, dynamic viscosity and fluid density. $A_{i j}$ represents the interface between $i$ and $j$ phases $(i, j=f$ (fluid), $b$ (biofilm), $s$ (solid), $h$ (NAPL)). We must keep in mind that, with the hypothesis of the quasi-steady-state processes of NAPL dissolution and bacterial growth, interface $A_{f i}$ between both regions is assumed to evolve with time but sufficiently slowly so that a no-slip condition can be adopted.

A non-boundary conforming numerical method is used to treat these dynamically changing pore-scale geometries due to NAPL dissolution and biofilm growth. Hereafter, the momentum equations are solved by using the immersed boundary-lattice Boltzmann (IB-LB) model presented in (Benioug et al., 2015) and already applied in (Benioug et al., 2017). A direct-forcing immersed boundary (IB) model originally derived by ( Mohd-Yusof , 1997) is coupled with the lattice Boltzmann method (LBM) to keep the fluid-solid interface sharp and maintain a direct control on numerical accuracy. Basically, it means that the fluid-biofilm-NAPL boundaries, which may evolve in time, are defined on a fixed grid mesh. A boundary force density term that acts as a forcing term is introduced into the momentum equation, Eq. (2), so that the no-slip condition is still satisfied on the immersed boundaries. For more details on the LB and IB methods, one can refer to (Succi, 2011, Khosronejad et al., 2011, De Rosis, 2014). To avoid excessive computational complexity, however, a piecewise linear interface construction (PLIC) approach is adopted so that the immersed boundaries still remain parallel to the facets of the cells. More details about these numerical methods and their validation can be found in (Benioug et al., 2015). 


\subsection{Solute transport}

Transport of dissolved hydrocarbon within this three-phase system is governed by the following conservation equations:

$$
\begin{array}{rlrl}
\frac{\partial c_{f}}{\partial t}+\nabla \cdot\left(\mathbf{u} c_{f}\right) & =\nabla \cdot\left(D_{f} \nabla c_{f}\right), & & \text { in the fluid phase } \\
\frac{\partial c_{b}}{\partial t} & =\nabla \cdot\left(D_{b} \nabla c_{b}\right)-R_{b}, & & \text { in the biofilm phase } \\
\frac{\partial c_{h}}{\partial t} & =0, & & \text { in the NAPL phase } \\
c_{f} & =K_{b f} c_{b}, & & \text { at } A_{f b} \\
D_{f} \nabla c_{f} \cdot \mathbf{n}_{f b} & =D_{b} \nabla c_{b} \cdot \mathbf{n}_{f b}, & & \text { at } A_{f b} \\
c_{f} & =K_{f h} c_{h}, & & \text { at } A_{f h} \\
D_{f} \nabla c_{f} \cdot \mathbf{n}_{f h} & =D_{h} \nabla c_{h} \cdot \mathbf{n}_{f h}, & & \text { at } A_{f h} \\
D_{f} \nabla c_{f} \cdot \mathbf{n}_{f h} & =-c_{h} \cdot \mathbf{w}_{f h} \cdot \mathbf{n}_{f h}, & & \text { at } A_{f h} \\
c_{b} & =K_{b h} c_{h}, & & \text { at } A_{b h} \\
D_{b} \nabla c_{b} \cdot \mathbf{n}_{b h} & =D_{h} \nabla c_{h} \cdot \mathbf{n}_{b h}, & & \text { at } A_{b h} \\
D_{b} \nabla c_{b} \cdot \mathbf{n}_{b h} & =-c_{h} \cdot \mathbf{w}_{b h} \cdot \mathbf{n}_{b h}, & & \text { at } A_{b h} \\
0 & =D_{f} \nabla c_{f} \cdot \mathbf{n}_{f s}, & & \text { at } A_{f s} \\
0 & =D_{b} \nabla c_{b} \cdot \mathbf{n}_{b s}, & \text { at } A_{b s} \\
0 & =D_{h} \nabla c_{h} \cdot \mathbf{n}_{h s}, & \text { at } A_{h s}
\end{array}
$$

where $c_{i}$ represents the hydrocarbon concentration within the phase $i, \mathbf{u}$ is the velocity field as predicted from Eqs. (1)-(5) and $\mathbf{n}_{i j}$ are the externals normal to the interfaces between phases $i$ and $j . D_{i}$ is the diffusion coefficient of hydrocarbon species in the phase $i . K_{i j}$ is the partition coefficient between the two phases $i$ and $j . R_{b}$ is the reaction term. $\mathbf{w}_{h j}$ is the local displacement velocity of the $A_{h j}$ interface. Note that $c_{h}$ is constant and corresponds to the density of the pure-phase NAPL compound.

Unlike the previous model (Benioug et al., 2017), it is important to emphasize that the computation of the transport equations is performed here in transient conditions. Indeed, since the transport of the dissolved hydrocarbon is directly related to the temporal evolution of the NAPL droplets, steady-state solution of the transport equations is never reached. However, we retain the hypothesis of quasi-steady-state transport processes with respect to the displacement kinetics of mobile interfaces (as well as the hypothesis of quasisteady-state of the flow) by sequentially solving the transport (Eqs. (6)-(15)), the NAPL dissolution (Eqs. (13)-(16)) and the bacterial growth equations.

The values of the partition coefficients $K_{f h}$ and $K_{b h}$ are different in order to take into account the 
presence of biosurfactants at the biofilm-NAPL interface. We usually have the correlation as:

$$
K_{f h} \leqslant K_{b h}
$$

It should also be noted that those three partition coefficients are not strictly independent. In order to satisfy the thermodynamic equilibrium condition of the system at rest, the equilibrium partition coefficient between the fluid and biofilm phases follows the relationship as:

$$
K_{b f}=\frac{K_{f h}}{K_{b h}}
$$

which can be derived from Eqs. $(9-14)$ as:

$$
\begin{aligned}
K_{b f} & =\frac{c_{f}}{c_{b}} \\
& =\frac{K_{f h} c_{h}}{K_{b h} c_{h}}
\end{aligned}
$$

Boundary conditions governing the evolution of the interface of the NAPL phase (Eqs. (13)-(16)) tend to link the interfacial diffusive flux to the displacement velocity $\mathbf{w}_{h j}$ of the interface $A_{h j}$, and are valid for the tracer transport (low concentration of dissolved hydrocarbon). The values of $\mathbf{w}_{f h}$ and $\mathbf{w}_{b h}$ are not necessarily identical between the fluid phase and the biofilm phase due to the variations in solubility. In the case of a NAPL droplet having an interface shared both by the biofilm and the fluid phases, this may theoretically lead to a deformation of the shape of the trapped droplet. However, Eqs. (13)-(16) do not take into account the surface tension that are supposed to counterbalance the deformation in theory. We will return in the next section on the approach to solve this problem and the numerical methods describing the dissolution of NAPL blobs.

The major difficulty in the spatial discretization of the mass conservation equations, Eqs. (6)-(15), is the presence of physical boundaries immersed in cells with 3 phases. In the case of the fluid-biofilm system studied in (Benioug et al., 2017), this problem was solved by a volume-of-fluid (VOF) type model based on a local equilibrium assumption between the two phases (also called "single-field model", see Marschall et al., 2012). In the presence of an additional NAPL phase, however, this assumption is getting more complicated. Considering the high concentration gradients existing in the field (the only sources of solute are from trapped droplets), the hydrodynamic conditions for which the NAPLs would be close to local equilibrium conditions with fluid and/or biofilm (even only in the vicinity of immersed boundaries) appear to be extremely limited. Based on this consideration, we opt for a reconstruction method of interfacial fluxes at the immersed boundaries (denoted by FV-reconstruction) (also referred to as the Cartesian cut-cell method with cell merging approach, see Ingram et al., 2003). The finite volume method (FVM) is used to discretize the equations. In practice, this consists of modifying the volume of the cell in the vicinity of the 
where $K_{i, j}$ is the partition coefficient between the phases of grid $i$ and $j$.

In the temporal discretization, the transport time step $t_{C F L}$ is determined from the velocities at the nodes based on the Courant conditions (or $C F L$ conditions for Courant-Friedrichs-Lewy) to avoid oscillation problems:

$$
t_{C F L}=\frac{C F L}{\max \left(U_{x}^{\max } / \Delta_{x}, U_{y}^{\max } / \Delta_{y}\right)}
$$




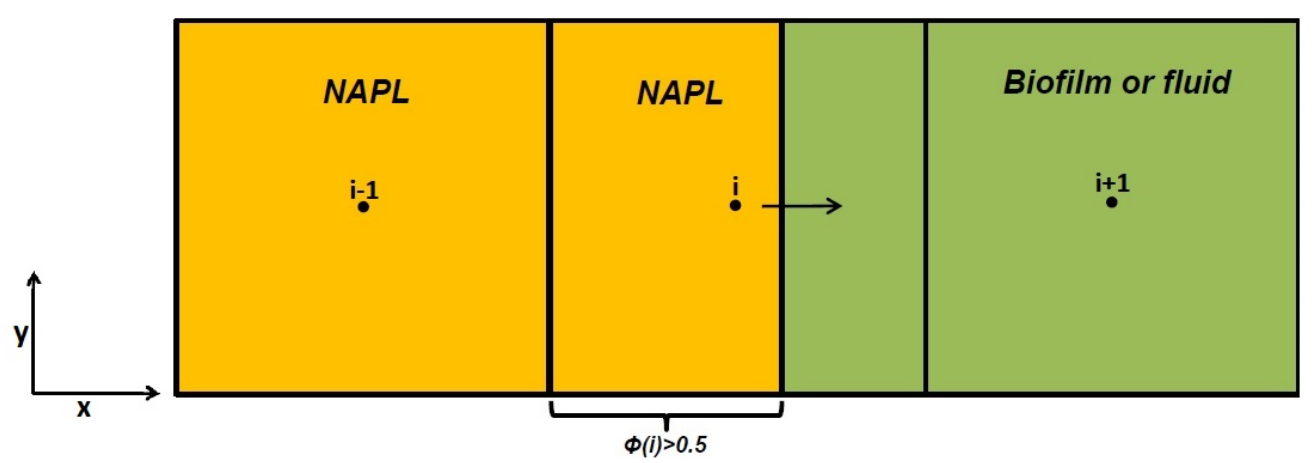

Figure 2: Extent of the cell $i+1$ (fluid/biofilm phase) and reduction of the cell $i$ (NAPL phase).

where $U_{x}^{\max }, U_{y}^{\max }$ are the maximum pore fluid velocities for the entire study domain in each direction, $\Delta_{x}$ and $\Delta_{y}$ are the grid sizes in each direction, and $C F L$ is a constant chosen as close as possible to 1 . Note that the net change in biomass concentration is calculated over a reference time step of growth, denoted by $t_{\text {growth }}$ and corresponding to an increase in biofilm volume fraction $\phi$ of 0.01 based on the maximum reaction rate $\mu_{\max }$.

\subsection{NAPL Dissolution}

As mentioned in the previous sections, differences in the solubility of the hydrocarbon in the presence of the fluid and biofilm phases will lead to variations in the interfacial fluxes that may be significant. In the case of a NAPL droplet in contact with these two phases, this may generate spatial changes in the dissolution rate over the periphery of the interface. Theoretically, this phenomenon should be compensated by the surface tension forces that have a stabilizing effect on the shape of the interface. If these forces are prevalent, the fluctuations in the dissolution rate will be distributed over the entire circumference so as to preserve a circular droplet. Otherwise, the differences between the dissolution rates will lead to a rupture of the interface that influences the formation of two new droplets. However, since we have deliberately chosen to ignore these surface tension effects, it is necessary to consider an alternative way in order to preserve a realistic modeling approach of the dissolution of the trapped droplet. A so-called uniform dissolution model has been developed.

This method distributes the dissolution uniformly around the droplet interface. In this way, the blob retains a circular shape throughout the dissolution process. Once the concentration field is calculated, the mass of NAPLs dissolved during the time $t_{C F L}, M_{b l o b}^{\text {diss }}$ is determined for each NAPL blob from Eqs. (13)(16). This dissolved quantity $M_{b l o b}^{\text {diss }}$ is obtained directly from the calculation of the diffusive fluxes at the 
interfaces:

$$
\begin{aligned}
M_{b l o b}^{\text {diss }} & =t_{C F L} \cdot \int_{A_{b l o b}}-c_{h} \mathbf{w}_{h} \cdot \mathbf{n}_{h} d A \\
& =t_{C F L} \cdot \int_{A_{b l o b}} D_{h} \nabla c_{h} \cdot \mathbf{n}_{h} d A
\end{aligned}
$$

where $A_{b l o b}$ is the exchange surface of the NAPL blob. After summing up the fluxes through each grid-cell boundary, we can determine the volume of the blob that is dissolved during the $t_{C F L}$ time step as well as the remaining volume $V_{b l o b}^{t+t_{C F L}}$ as:

$$
\begin{aligned}
V_{b l o b}^{\text {diss }} & =\frac{M_{b l o b}^{\text {diss }}}{c_{h}} \\
V_{b l o b}^{t+t_{C F L}} & =V_{b l o b}^{t}-V_{b l o b}^{\text {diss }}
\end{aligned}
$$

where $V_{b l o b}^{t}$ is the volume of the NAPL blob at the time $t$ (before the dissolution step) and $V_{b l o b}^{\text {diss }}$ is the volume dissolved during the time step $t_{C F L}$. From the blob's original circular shape, it is then sufficient to update its radius $R^{t+t_{C F L}}$ from the following relation:

$$
R^{t+t_{C F L}}=\sqrt{\frac{V_{b l o b}^{t+t_{C F L}}}{\pi}} \text { in } 2 D
$$

\subsection{Bacterial growth model and inhibitory effect}

The time evolution of the biomass concentration $\rho_{\text {bio }}$ is governed by the Haldane model (Saravanan et al., 2008), which is developed to study the impact of NAPL toxicity on biomass. The Haldane model is widely used to represent the influence of inhibition effect on the kinetics of the bacteria growth, including the conditions with the presence of a biodegradable substrate but toxic for high concentrations (Kumar et al., 2005). The reaction term $R_{b}$ in Eq. (7) is written in the form of:

$$
R_{b}=\rho_{b i o} \mu_{\max } \frac{C}{C+K+\frac{C^{2}}{K_{i}}},
$$

and the bacterial growth can be calculated from the following equation:

$$
\frac{\partial \rho_{b i o}}{\partial t}=F_{\lambda} \rho_{b i o} \mu \frac{C}{C+K+\frac{C^{2}}{K_{i}}}-k_{d} \rho_{b i o}
$$

where $\rho_{b i o}, \mu, K$ are, respectively, the biomass concentration, the maximum reaction rate and the halfsaturation constant. $F_{\lambda}$ is the stoichiometric coefficient of the biological reaction, $k_{d}$ is the extinction 
coefficient of the bacteria and $K_{i}$ is the inhibition coefficient. For illustrative purposes, Fig. 3 compares the evolution of the reaction rate $R_{b}$ predicted by the Monod and Haldane models as a function of substrate concentration. As a reminder, Monod kinetics is classically described by:

$$
R_{b}=\rho_{b i o} \mu_{\max } \frac{C}{C+K},
$$

We can see the impact of the inhibition effect by the decrease of the reaction rate when the concentration is increased (Fig. 3).

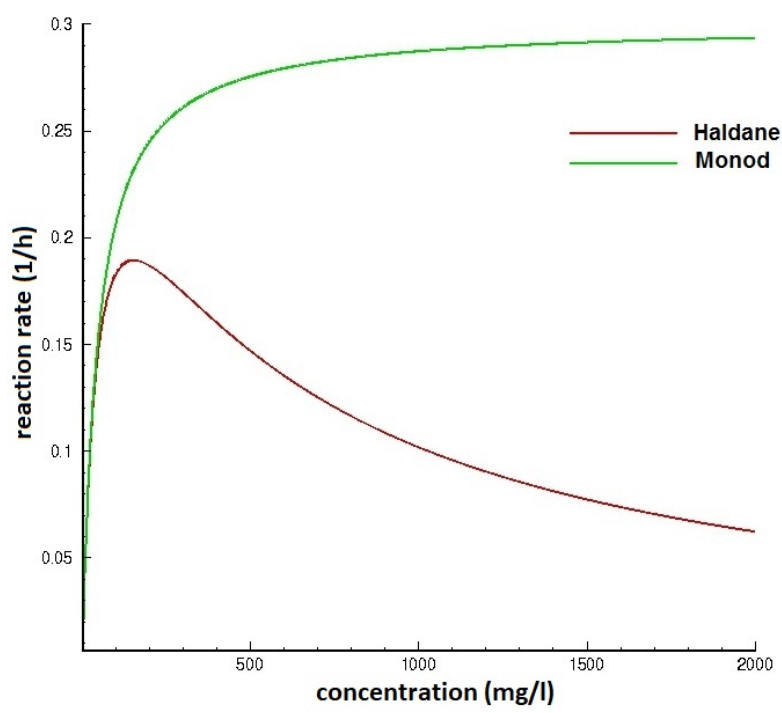

Figure 3: Reaction rate as a function of substrate concentration predicted by the Monod and Haldane model $\left(\mu=0.3 h^{-1}\right.$, $\left.K=44.9 \mathrm{mg} / \mathrm{l}, K_{i}=525 \mathrm{mg} / \mathrm{l}\right)$

The biomass concentration $\rho_{\text {bio }}$ calculated at each time step from Eq. (34) is then used to determine the value of the biofilm volume fraction $\phi$ per cell (the biomass density is supposed to be constant in the whole domain). The net change in biomass concentration is calculated over a reference time step of growth $\left(t_{\text {growth }}\right)$ and corresponding to an increase in $\phi$ of 0.01 based on the maximum reaction rate $\mu_{\max }$. As soon as $\rho_{b i o}$ exceeds locally, within a biofilm cell, an arbitrary value denoted by $\rho_{\text {biomax }}$ (corresponding to a value of $\phi=1$ ) defined as the biomass density is distributed spatially by using the cellular automata model. The algorithm introduced in (Benioug et al., 2017) will be briefly explained here. Basically, in each grid cell $(x, y)$, as soon as $\phi(x, y)>1$ (i.e., $\left.\rho_{\text {bio }}(x, y)>\rho_{\text {biomax }}\right)$, the excess of biomass $\Delta \rho_{\text {bio }}=\rho_{\text {bio }}(x, y)-\rho_{\text {biomax }}$ is moved towards an adjacent fluid cell, randomly chosen with equal probability, and this cell becomes a partially-filled cell with a volume fraction of $\phi=\frac{\Delta \rho_{b i o}}{\rho_{\text {biomax }}}$. This algorithm is repeated for each cell in turn until all the excess biomass is redistributed.

Finally, the general algorithm of our model including fluid flow, chemical species transport, biofilm growth and extinction, and NAPL dissolution is illustrated in Fig. 4. Note that the biofilm growth model has been 
previously validated from qualitative comparison with benchmark cases of the literature in (Benioug et al., 2017).

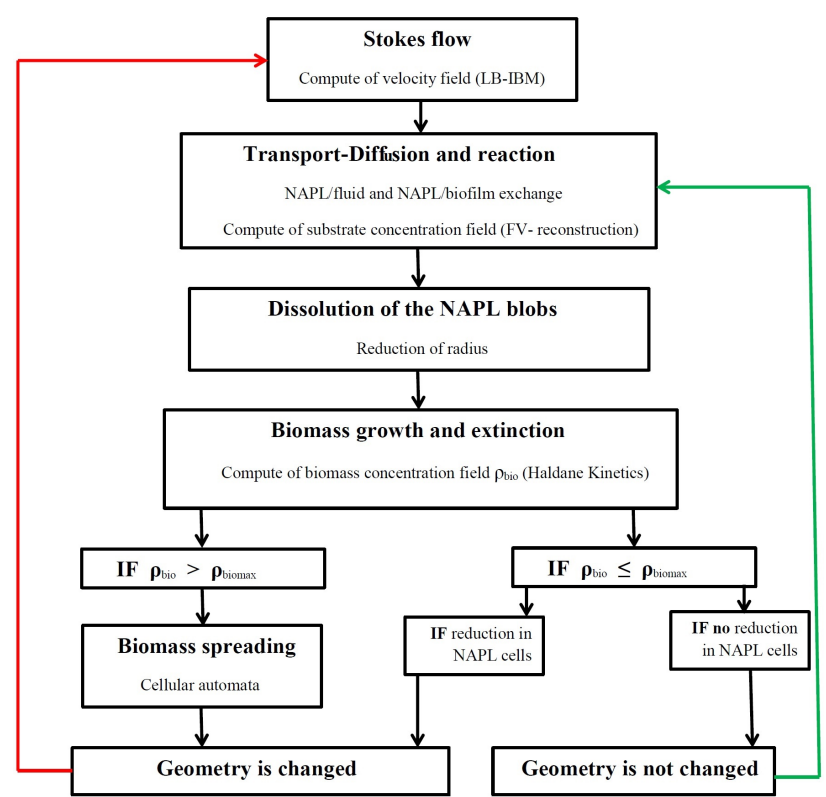

Figure 4: Algorithm of the dissolution and biodegradation model of a hydrocarbon phase at the pore scale

\section{Results and discussion}

In order to demonstrate the capability of the current model in simulating the complex and dynamic processes in porous media (e.g. NAPL blobs reduction, biofilm growth), as well as its predictability to study the impact of the main bio-physico-chemical mechanisms on the dissolution of the residual hydrocarbon phase, we test our dissolution model under various hydrodynamic conditions. The influence of different parameters (such as Péclet number Pe, Damköhler number $D a$, heterogeneity, etc.) will thus be tested separately.

To facilitate the analysis and the interpretation of results, numerical data will be reported in a dimensionless form as described below:

$$
\begin{array}{r}
x^{\prime}=\frac{x}{l}, y^{\prime}=\frac{y}{l}, t^{\prime}=t \frac{D_{f}}{l^{2}}, c_{i}^{\prime}=\frac{c_{i}}{c_{h}}, \quad(i=f, b, h) \\
p^{\prime}=\frac{p l}{\mu_{f} u_{m o y}}, \mathbf{u}^{\prime}=\frac{\mathbf{u}}{u_{\text {moy }}}, \rho_{b i o}^{\prime}=\frac{\rho_{b i o}}{\rho_{\text {biomax }}}, D_{i}^{\prime}=\frac{D_{i}}{D_{f}}, \\
K^{\prime}=\frac{K}{c_{h}}, K_{i}^{\prime}=\frac{K_{i}}{c_{h}}, \mu^{\prime}=\mu \frac{l^{2}}{D_{f}}, k_{d}^{\prime}=k_{d} \frac{l^{2}}{D_{f}}, \mathbf{w}_{i j}^{\prime}=\frac{\mathbf{w}_{i j} l}{D_{f}}
\end{array}
$$

where $\rho_{\text {biomax }}, u_{\text {moy }}$ and $l$ respectively represents the maximum biomass concentration, the averaged fluid velocity and the characteristic length of the medium. We also introduce the following dimensionless numbers, 
$P e$ and $D a$ that characterize mass transport and bacterial reactivity, defined as:

$$
\begin{aligned}
\text { Péclet number: } P e & =\frac{u_{\text {moy }} \cdot l}{D_{f}} \\
\text { Damköhler number: } D a & =\frac{\mu_{\max } \cdot l^{2} \cdot \rho_{\text {biomax }}}{D_{f} K}=\frac{\mu_{\text {max }}^{\prime}}{K^{\prime}} \Gamma \text { with } \Gamma=\frac{\rho_{\text {biomax }}}{c_{h}}
\end{aligned}
$$

The influence of fluid flow and substrate consumption are then investigated through different values attributed to the dimensionless Péclet and Damköhler numbers.

\subsection{NAPL dissolution within a single pore}

As the first step, we need to test our model by simulating the dissolution process with and without biodegradation in a simple pore structure - a single NAPL droplet in a narrow channel. The computational domain $\Omega=[0, l x] \times[0, l y]\left(l x \times l y=30 \times 10 \mathrm{~mm}^{2}\right)$ is discretized with uniform mesh $((N x-1) \times(N y-1))$ with a resolution of $\Delta x=\frac{l x}{N x-1}=\Delta y=\frac{l y}{N y-1}$, where $N x$ and $N y$ are the number of nodes along the $(x)$ and $(y)$ directions. A velocity profile is imposed at the inlet $\left(x^{\prime}=1\right)$; while a constant pressure at the outlet $\left(x^{\prime}=3\right)$. No slip-boundary conditions are introduced at the wall boundaries $\left(y^{\prime}=0\right.$ and $\left.y^{\prime}=1\right)$. The NAPL droplet is in circular shape with a radius of 0.21 , with its center located at the coordinates $(1,0.5)$. The bacteria corresponding to $0.08 \%$ of total volume are randomly distributed on the NAPL droplet surface as shown in Fig. 5a (biomass is presented here in white). The input data are summarized in Table 1. Figures 5b-5c show the NAPL droplet dissolution with and without biodegradation. A reduction over time in the size of the NAPL droplet is captured while its circular shape being maintained, which is in agreement with our uniform dissolution approach. If the NAPL blob gradually decreases in both cases, for biotic and abiotic conditions, its decrease is much faster in the presence of bacteria. At the time $t^{\prime}=500$, the volume fraction of the NAPL is equal to 0.16 in abiotic condition, while it is equal to 0.1 in biotic conditions. This acceleration of NAPL degradation in the presence of bacteria is due to the biosurfactant-induced change of the partitioning coefficient on the surface of the contaminant droplet.

From the above attempt, our model is able to correctly describe the physics of the NAPL dissolution. However, as the dissolution process depends on the velocity field, we need to assess the mesh-dependency of our numerical results. Three other meshes are considered for this purpose $(450 \times 150,225 \times 75$, and $25 \times 75)$. The decrease of the NAPL blob volume over time is illustrated in Figure 5d, which is almost identical for the meshes $(450 \times 150,300 \times 100,225 \times 75)$ proving our numerical simulations are mesh independent. When continuing to degrade the mesh size, the surface of the NAPL blob which drives the dissolution rate is too overestimated in spite of using immersed boundaries. However, even for the mesh $(75 \times 25)$, with less than 10 cells along the initial diameter, the numerical error on the total dissolution time is lower than $10 \%$ ( $t^{\prime}=2400$ against $t^{\prime}=2650$ for the refined solution). This comparison guided our spatial discretization process and in the following simulations, we do not go below this lower limit for mesh discretization (i.e., 10 cells along the initial NAPL blob diameter). 
Table 1: Physical and biological parameters used in the single pore simulations.

\begin{tabular}{l|l|l}
\hline Parameter & $\begin{array}{l}\text { physical/biological } \\
\text { value }\end{array}$ & dimensionless value \\
\hline$l x$ & $30 \mathrm{~mm}$ & $l x^{\prime}=3$ \\
$l y$ & $10 \mathrm{~mm}$ & $l y^{\prime}=1$ \\
$N x$ & 301 & \\
$N y$ & 101 & \\
$c_{h}$ & $800 \mathrm{mg} / \mathrm{l}$ & $c_{h}^{\prime}=1$ \\
$C_{e q}$ & $0.48 \mathrm{mg} / \mathrm{l}$ & $C_{e q}^{\prime}=6 \times 10^{-4}$ \\
$D_{f}$ & $10^{-9} \mathrm{~m}^{2} / \mathrm{s}$ & $D_{f}^{\prime}=1$ \\
$D_{b}$ & $0.25 \times 10^{-9} \mathrm{~m}^{2} / \mathrm{s}$ & $D_{b}^{\prime}=0.25$ \\
$K_{f h}$ & - & $6 \times 10^{-4}$ \\
Péclet number, $(P e)$ & - & 0.3 \\
\hline$K$ & $0.24 \mathrm{mg} / \mathrm{l}$ & $K^{\prime}=3 \times 10^{-4}$ \\
$k_{d}$ & $2 \times 10^{-14} \mathrm{~s}$ & $k_{d}^{\prime}=2 \times 10^{-7}$ \\
$K_{i}$ & $0.64 \mathrm{mg} / \mathrm{l}$ & $K_{i}^{\prime}=8 \times 10^{-4}$ \\
$K_{b h}$ & - & $6 \times 10^{-3}$ \\
$F_{\lambda}$ & - & 0.3 \\
Damköhler number, $(D a)$ & - & $0.1,10$ \\
\hline
\end{tabular}

\subsection{NAPL dissolution in porous media}

We consider now a representative volume of a 2D porous medium (Fig. 6). The domain has a size of $l x \times l y=12 \times 12 \mathrm{~mm}^{2}$ with an initial porosity $\epsilon_{0}$ of 0.67 and an initial NAPL saturation $s_{0}=4.2 \%$. The pore throat characteristic length, $l_{f}$, is estimated to be $0.3 \mathrm{~mm}$. An initial velocity profile is imposed at the inlet of the domain (i.e., at $x^{\prime}=0$ ) while the pressure is kept constant at the outlet (i.e., at $x^{\prime}=1$ ). Periodic conditions are introduced at the boundaries at $y^{\prime}=0$ and $y^{\prime}=1$.

\subsubsection{NAPL dissolution without biodegradation}

In this section, we examine the influence of the spatial distribution of NAPL droplets and the flow velocity on the dissolution within the porous medium. We initially consider three NAPL droplets trapped in the pores of the medium as shown in Figure 6. At this point, there is no bacterial population that can biodegrade these organic compounds. The values of input data used for this simulation are presented in Table 2 . 

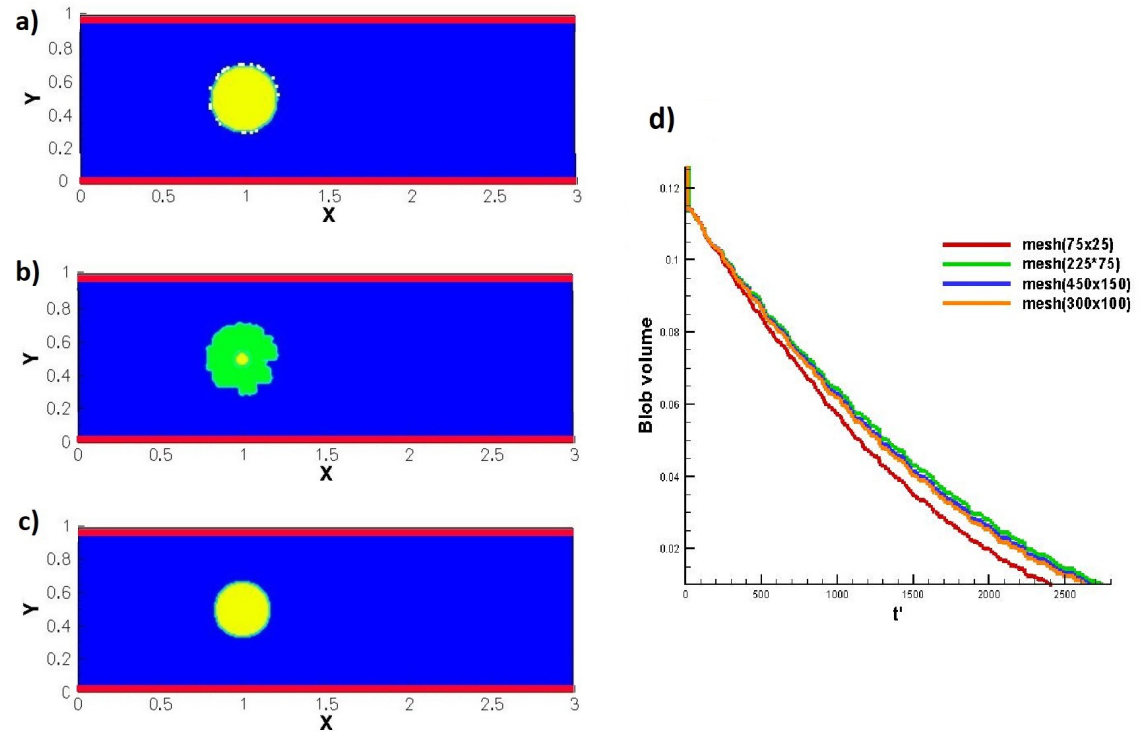

Figure 5: a) The initial geometry of the computational domain: red - solid phase, blue - fluid phase, yellow - NAPL, blue cyan - NAPL/fluid grids and white - biofilm; b) Geometry of the medium at $t^{\prime}=500$ with the presence of the biofilm (in green); c) Geometry of the medium at $t^{\prime}=500$ without biofilm; d) Temporal variation of the NAPL blob volume.

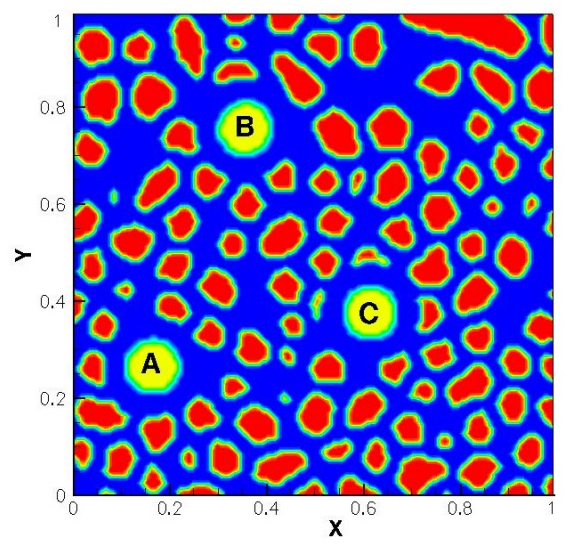

Figure 6: The initial geometry of the computational domain: red - solid phase, blue - fluid phase, yellow - NAPL and blue cyan - NAPL/fluid grids. 
Table 2: Physical parameters used in the simulations.

\begin{tabular}{l|l|l}
\hline Parameter & Physical value & $\begin{array}{l}\text { Dimensionless } \\
\text { value }\end{array}$ \\
\hline $\begin{array}{l}\text { Length of the domain, } l x \\
\text { Width of the domain, } l y\end{array}$ & $12 \mathrm{~mm}$ & $l x^{\prime}=1$ \\
Number of mesh cells in $x$ & 116 & $l y^{\prime}=1$ \\
direction, $N x$ & & \\
Number of mesh cells in $y$ & 116 & \\
direction, Ny & & \\
$c_{h}$ & $800 \mathrm{mg} / l$ & $c_{h}^{\prime}=1$ \\
$D_{f}$ & $0.25 \times 10^{-9} \mathrm{~m}^{2} / \mathrm{s}$ & $D_{f}^{\prime}=1$ \\
$D_{b}$ & - & $D_{b}^{\prime}=0.25$ \\
$K_{f h}$ & & $6 \times 10^{-3}$ \\
NAPL blobs location & - & $A(0.27,0.17)$, \\
$\left(x_{c e n t e r}^{\prime}, y_{c e n t e r}^{\prime}\right)$ & & $C(0.76,0.36)$, \\
& & 20 \\
\hline Péclet number, $(P e)$ & - & \\
\hline
\end{tabular}

\section{Influence of the blobs distribution}

We consider the same radius for three NAPL blobs $R_{A}=R_{B}=R_{C}=0.055$ (dimensionless, equal to their physical values divided by the length of the domain). Results illustrated in Figs. 7b-7d show reasonable behavior of the flow. The velocity field is in agreement with the assumptions imposed: the upstream fluid flow bypass the trapped droplets of NAPL behaved as obstacles and form perturbations behind. A consistent pattern is also observed for the concentration fields (Figs. 7a-7c): (i) the hydrocarbon plume resulting from the dissolution is well entrained by the flow downstream of the NAPLs and follows the preferential flow paths between the solid grains and (ii) the plume of dissolved contaminant reabsorbs as the dissolution of the droplets progresses. Finally, NAPL blobs, which gradually decrease over time, retain in circular shapes, as assumed by our model. In other words, the mechanisms behind the dissolution of the NAPL are correctly taken into account by our model demonstrated by reasonable and consistent flow/concentration field results.

Figure 7e shows a nearly linear correlation between the droplet radius and time, which is in agreement with the experimental results obtained by (Kennedy and Lennox, 1997) and ( Poesio and al,, 2009). This relationship reveals that the dissolution kinetics are independent of the size of the NAPL droplets, at least with the numerical conditions imposed. Figure $7 \mathrm{f}$ confirms such result by reducing the volume of the dissolved NAPL in a quadratic way. This nonlinear decrease is obviously related to the reduction of the exchange 


\section{$t^{\prime}=5$}
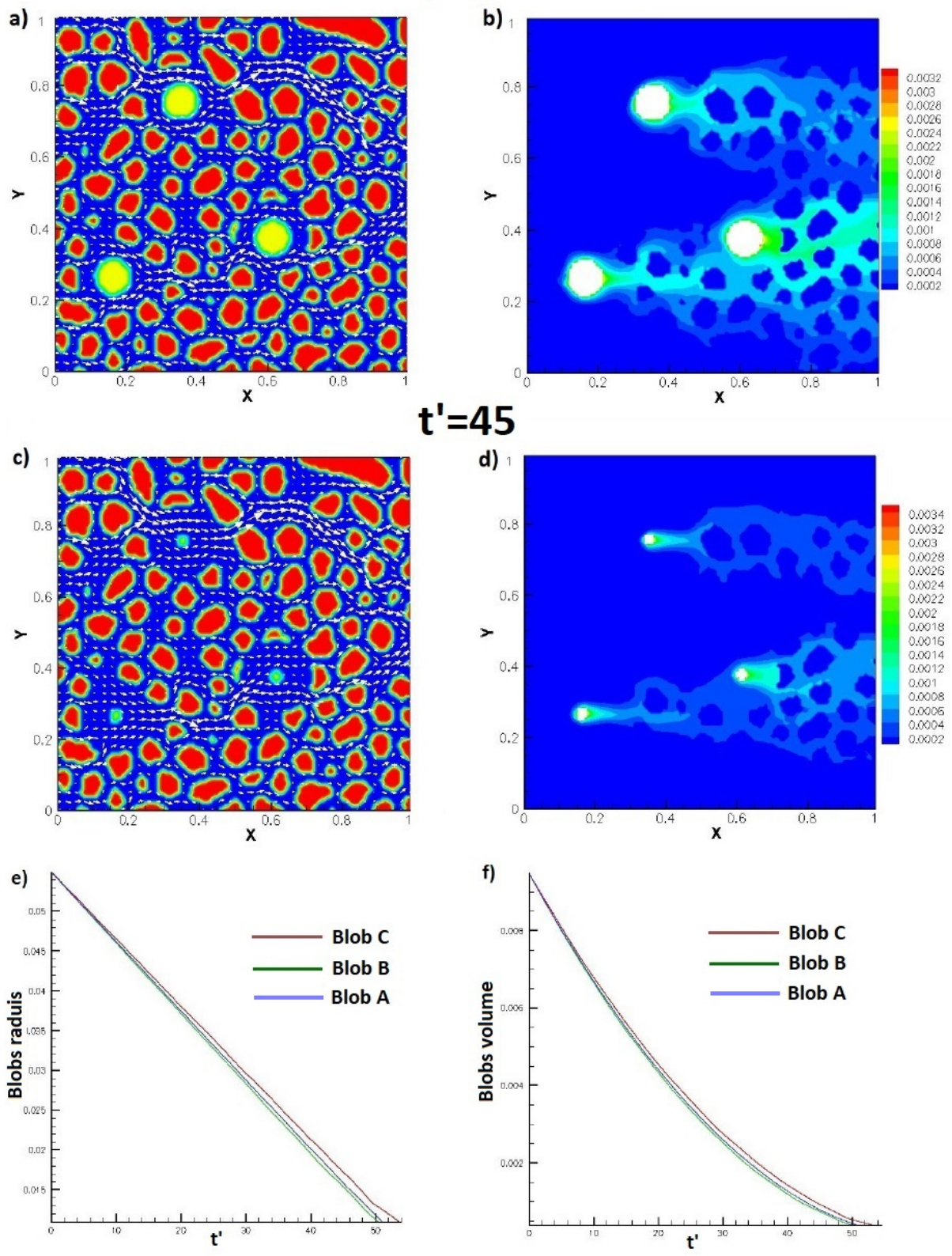

Figure 7: (a, c) Hydrocarbon concentration, NAPL blobs are white; (b, d) Geometry of the porous medium and the velocity field. a), b) at $t^{\prime}=5$; c), d) at $t^{\prime}=45$; e) Temporal variation of NAPL blobs radius; f) Temporal variation of NAPL blobs volume. 
surface between the NAPL blob and the surrounding fluid that leads to a decrease in the interfacial fluxes exchanged between two phases, which also agrees with the observations of (Kennedy and Lennox, 1997).

On the other hand, if each NAPL blob evolves constantly over time, variations in the rate of dissolution between the droplets are analyzed. Figures $7 \mathrm{e}-7 \mathrm{f}$ show that droplet $B$ dissolves a bit more rapidly while droplet A dissolves before droplet $C$. This disparity is justified by their spatial distribution and the establishment of preferential flow paths linked to the heterogeneity of the medium at the pore scale. Indeed, since the blob $\mathrm{C}$ is located downstream of the dissolution plume of blob A, dissolved contaminant is advected by the bulk fluid flow in the vicinity of blob C. This plume channeling generates low concentration gradients around this blob and hence a substantial decrease of the dissolution rate (Figs. 7c-7e). In contrast, droplet $B$ is trapped in a macropore where the flow velocities are greater, which leads to an increase in the concentration gradient and therefore in the flow exchanged. These results are also in agreement with the experimental observations in the literature (Kennedy and Lennox, 1997, Imhoff et al., 1994).

\section{Influence of the flow velocity}

Flow velocity plays a non-negligible role in the dissolution of the NAPL droplets as it directly impacts the mass transfer to the bulk fluid. To investigate the impact of flow velocity, we compare our simulated results under two flow rates. The same simulation setup is kept here as in the previous section except for two parameters, namely the size of the droplets and the Péclet number. The radius of the NAPL droplets are now: $R_{A}=0.05, R_{B}=0.055, R_{C}=0.06$. Figure 8 shows the dissolution of these blobs for two Péclet numbers, respectively $P e=0.5$ and $P e=10$ and at two different moments, $t^{\prime}=5$ and $t^{\prime}=51$.

A closer observation of the concentration fields indicates that the spreading of the NAPL plume is strongly influenced by the Péclet number ( $P e=10$ : Figs. 8b, 8f; $P e=0.5$ : Figs. 8d, 8h). When the number of Péclet increases, the lateral extension of the plume is reduced. From a macroscopic point of view, this classical behavior translates into in an increase in the dispersion coefficient with the Péclet number (Powers et al., 1992, Ahmadi et al., 2001). In other words, the increase in the Péclet number involves strong concentration gradients around the NAPL droplets and thus an increase of the dissolved NAPL flux. This increase obviously leads to a more rapid dissolution of the residual saturation zones (Figs. 8a, 8e). As we can see, at $t^{\prime}=51$, the dissolution of the NAPL droplets is practically completed for $P e=10$ (Fig. 8e) while all three droplets are still visible for $P e=0.5$ (Fig. 8g). For low Péclet numbers, indeed, the diffusive effects are still strongly predominant. The exchanges are limited by the external mass transfer and the dissolution of the trapped droplets is relatively slower (Figs. 8c, 8g).

Upscaling from the pore-scale to Darcy-scale (over the entire porous sample), Fig. 9 shows the reduction of the total volume fraction of the trapped NAPLs over time for both flow velocities. An approximately $6 \%$ increase in the apparent rate of dissolution was observed for $P e=10$ compared to $P e=0.5$. This increase may seem small given the increase in velocity by a factor of 20 but it may be explained by the very low 

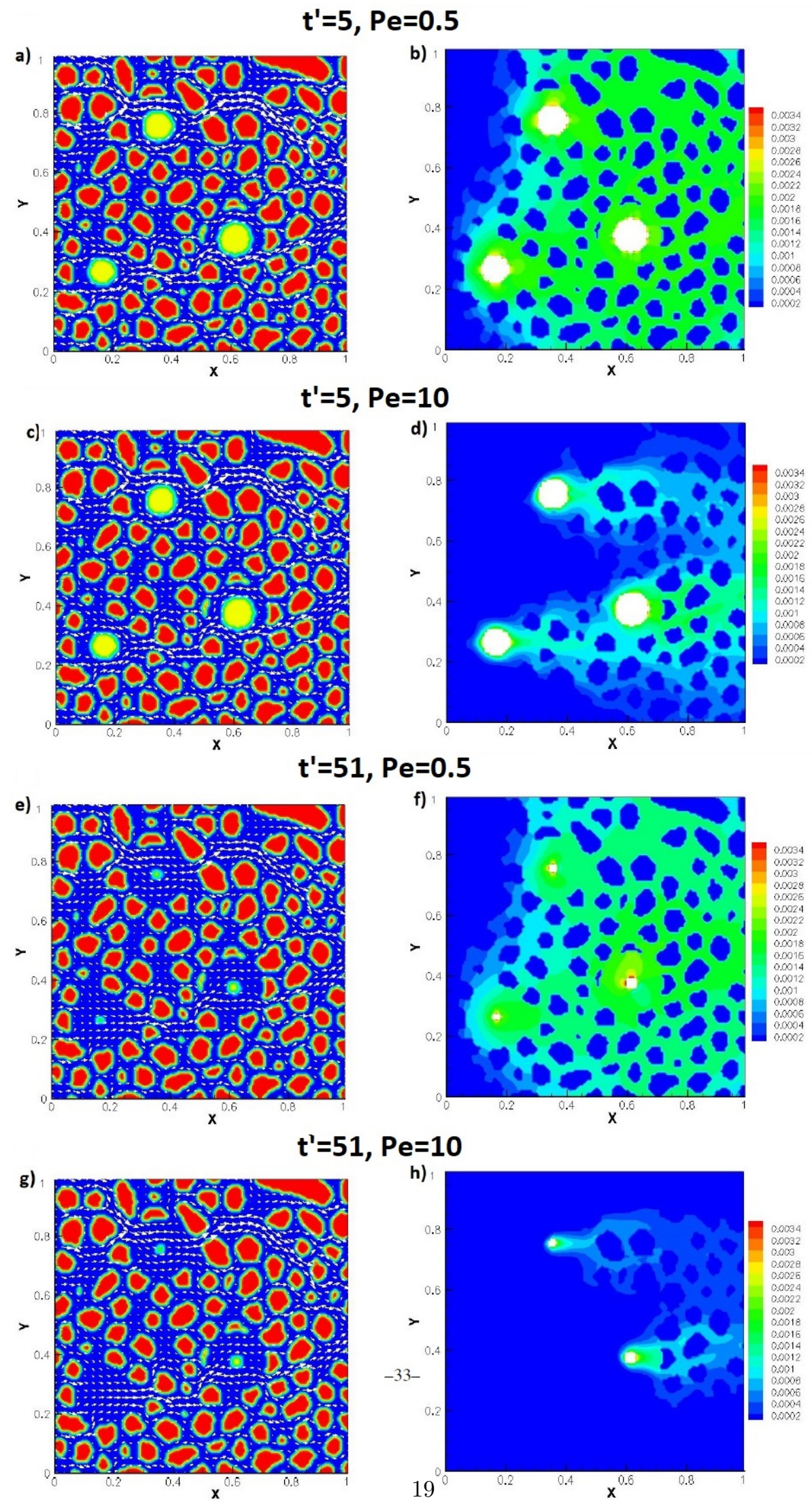

Figure 8: Evolution of the transport processes (a, c, e, g) and associated concentration fields (b, d, f, h) at two different times for two Péclet numbers: a), b) $t^{\prime}=5-P e=10 ;$ c), d) $t^{\prime}=5-P e=0.5 ;$ e), f) $t^{\prime}=51-P e=10 ;$ g), h) $t^{\prime}=51-P e=0.5$. 
saturation of NAPLs initially present in the medium $\left(s_{0}=4.2 \%\right)$. Note that (Ahmadi et al., 2001) obtained comparable results in terms of order of magnitude for mass transfer coefficient variations from 2D unit cell calculations (increase of the exchange coefficient by a factor of about $10 \%$ for the same range of variation of $P e$ when the NAPL saturation is less than $5 \%$ ).

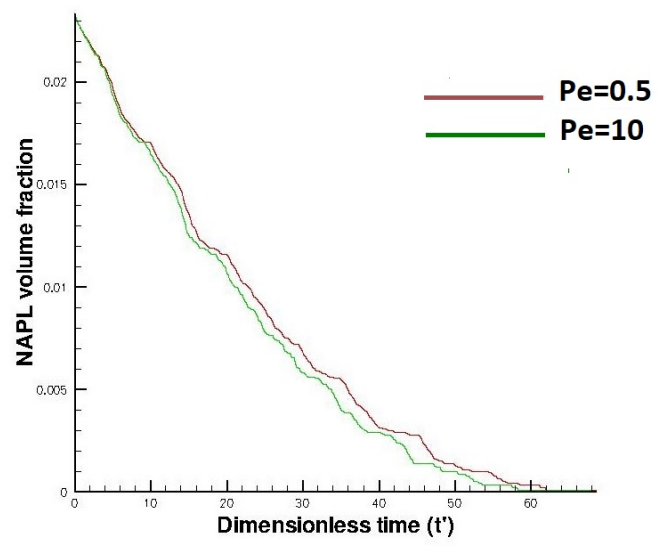

Figure 9: Temporal variation of the NAPL volume fraction for two Péclet numbers: $P e=10, P e=0.5$

\subsubsection{Bio-enhanced NAPL dissolution and biofilm growth}

In this section, the same porous media domain is initially injected with bacteria capable of biodegrading the hydrocarbon. The distribution of the oil phase is similar to that of the previous section. Bacteria, present in sessile form, initially occupy $13.7 \%$ of the pore volume, distributed randomly on the solid surfaces. Simulation parameters are shown in Table 3. The color scale used to represent the different grids is detailed in Fig. 10h and will be kept for the rest of the study. It should be noted here that we have represented the fluid/biofilm immersed cells as biofilm cells in order to limit the number of colors used and to facilitate the readability of the figures.

First, we considered cases with relatively low Damköhler and Péclet numbers, i.e., $D a=20$ et $P e=0.5$. Moreover, in order to study the influence of the solubility increase of dissolved contaminants in contact with bacteria, we have taken a high coefficient of inhibition, i.e., $K_{i}^{\prime}=2 \times 10^{-3}$. This high value of $K_{i}$ allows us to eliminate the toxicity effects of NAPLs so that the bacteria can grow even in the presence of an excess of substrate. In other words, this hypothesis favors bacterial growth in the vicinity (or even in contact) of the NAPL blobs.

Figures 10a-10d indicate that the biofilm grows preferentially near the NAPL blobs. Conversely, few biofilm cells develop far away from the residual NAPL droplets. Over time, the volume of biomass increases and the biofilm approaches the NAPL blobs until reaching the interface. This preferential growth clearly reflects bacterial growth where the substrate concentration is maximum (NAPL blobs are the only substrate 

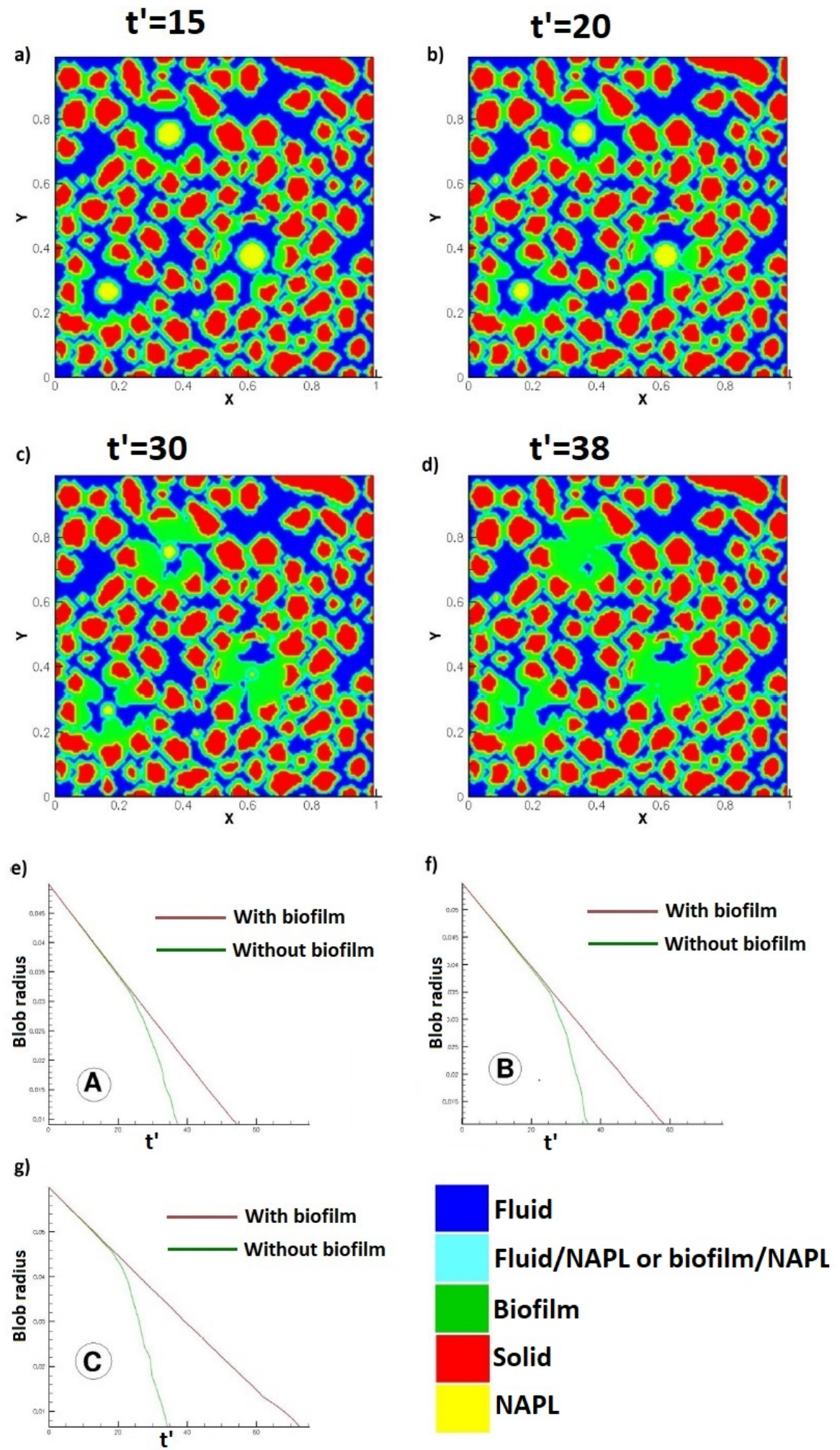

Figure 10: Evolution of the transport processes for $\left.\left.\left.D a=20(\mathrm{a}, \mathrm{b}, \mathrm{c}, \mathrm{d}): \mathrm{a}) t^{\prime}=15, \mathrm{~b}\right) t^{\prime}=20, \mathrm{c}\right) t^{\prime}=30, \mathrm{~d}\right) t^{\prime}=38$; temporal variation of blobs radius with/without biofilm (e, f, g): e) blob A, f) blob B, g) blob C; h) color scale of grid types. 
Table 3: Physical and biological parameter values used in the simulations

\begin{tabular}{l|l|l}
\hline Parameter & $\begin{array}{l}\text { physical/biological } \\
\text { value }\end{array}$ & dimensionless value \\
\hline$l x$ & $12 \mathrm{~mm}$ & $l x^{\prime}=1$ \\
$l y$ & $12 \mathrm{~mm}$ & $l y^{\prime}=1$ \\
$N x$ & 116 & \\
$N y$ & 116 & \\
$c_{h}$ & $800 \mathrm{mg} / l$ & $c_{h}^{\prime}=1$ \\
$D_{f}$ & $10^{-9} \mathrm{~m}^{2} / \mathrm{s}$ & $D_{f}^{\prime}=1$ \\
$D_{b}$ & $0.25 \times 10^{-9} \mathrm{~m}^{2} / \mathrm{s}$ & $D_{b}^{\prime}=0.25$ \\
$K_{f h}$ & - & $6 \times 10^{-3}$ \\
Péclet number, $(P e)$ & - & 0.5 \\
\hline Damköhler number, $(\mathrm{Da})$ & - & 20 \\
$K$ & $0.8 \mathrm{mg} / \mathrm{l}$ & $K^{\prime}=10^{-3}$ \\
$k_{d}$ & $2 \times 10^{-14} \mathrm{~s}$ & $k_{d}^{\prime}=2 \times 10^{-7}$ \\
$K_{i}$ & $1.6 \mathrm{mg} / \mathrm{l}$ & $K_{i}^{\prime}=2 \times 10^{-3}$ \\
$K_{b h}$ & - & $6 \times 10^{-2}$ \\
$F_{\lambda}$ & - & 0.3 \\
\hline
\end{tabular}

source). Taking into account both the initial spatial distribution of NAPL and biofilm, and preferential flows (highlighted in the previous paragraph), droplet $\mathrm{C}$ is the first blob reached by the bacterial development, i.e., towards $t^{\prime}=15$, whereas droplet B is the last (at $t^{\prime}=20$ ). Once the biofilm is in contact with NAPL, the dissolution process accelerates under the effect of biosurfactants that increase the contaminant solubility. It is thus observed that droplet $\mathrm{C}$, although it is the widest $\left(R_{C}=0.06\right)$, is dissolved first.

In order to confirm this hypothesis, we compare in Figs. 10e-10g the evolution of the NAPL blob radius with and without the presence of the bacteria. We first note that, before the contact between NAPL and biofilm, dissolution kinetics are almost the same in biotic and abiotic conditions. However, once the biofilm adhere to the surface of the droplet of NAPL, the dissolution rate increases significantly. On one hand, the contact with the bacteria favors the solubility of the hydrocarbon due to the surfactants. On the other hand, the bacterial assimilation of the organic contaminant accentuates the concentration gradients and therefore the interfacial fluxes. This so-called 'bio-enhanced dissolution' phenomenon has been already identified by previous studies (Seagren et al., 1994, Yang and L. McCarty, 2000).

Reactions induced by bacteria can impact the dissolution rate of NAPLs via the increase of the biomass concentration which plays an essential role in the spatial and temporal evolution of the pore structure. 
Knowing the most influential parameters on the change of this rate of dissolution constitutes an important step in modeling the NAPLs. Thus, in the following sections, our interest will be focused on the influence of the Damköhler number and the coefficient of inhibition on the dissolution process.

\section{Impact of Damköhler number}

In this paragraph we keep the same setup as used previously (Tab. 3), with the exception of the Damköhler number set at $D a=50$. This high value translates into an increase in the reaction kinetics and therefore in the amount of biodegradated NAPL compared to the results obtained at $D a=20$. It should also favor an increased biomass production in the immediate vicinity of NAPL blobs.

Our simulated results, illustrated in Figures 11a-11d, confirm such hypothesis. A more rapid growth of biomass is shown compared to the results observed at $D a=20$ in Fig. 10. In fact, the blobs of NAPL are in contact with the bacterial filaments much more quickly, i.e., at $t^{\prime}=7$ for $D a=50$ against $t^{\prime}=15$ for $D a=20$. Similarly, at $t^{\prime}=24$ (Fig. 11c), practically all the blobs are dissolved (against $t^{\prime}=38$ for $D a=20)$. This behavior is also verified in Figs. 11d-11f where we compared the evolution of the different blobs for two Damköhler numbers. The dissolution is faster when $D a$ increases for all droplets.

As long as the biofilm is not in contact with the NAPL droplets, the increase of the dissolution rate with Damköhler number is low. The gap is strongly accentuated, however, with the biofilm adhesion to the NAPL blobs. Biodegradation, which is conditioned by the $D a$ value, then plays a preponderant role in the dissolution process by increasing the concentration gradient and consequently the dissolved NAPL fluxes.

However, the influence of structural heterogeneity seems to dissipate with the increase of $D a$. Indeed, the dissolution of the droplet C, which was favored by the preferential flow effects at low $D a$ (Fig. 10), seems to be somewhat delayed here. As it can be seen in Fig. 11a, the bio-clogging of the pores in the vicinity of the trapped droplets plays as barriers to the flow and leads to a reduction of the velocity (and thus the local Péclet number) near the water/NAPL interface. Too high values of Damköhler number could thus have a paradoxical effect and slow down the dissolution process instead of accelerating it by bio-clogging the pores at the entrance of the porous medium and thus limiting the availability of trapped NAPL droplets.

\section{Impact of toxicity}

The impact of NAPL toxicity on bacterial growth is taken into account in our model through the use of Haldane kinetics (Saravanan et al., 2008). The reaction rate is thus no longer only controlled by the Damköhler number and the half-saturation constant as in the Monod kinetics model but also by the inhibition coefficient $K_{i}$ which plays an essential role in the bacterial growth/decay when the tolerance threshold for the substrate concentration is exceeded. In this section, we use the same data as those summarized in Table 3 , with the exception of the values of the inhibition $K_{i}$ and extinction $k_{d}$ coefficients. We first keep the value of the extinction coefficient as constant $\left(k_{d}^{\prime}=10^{-7}\right)$ and study the influence of the inhibition coefficient. 

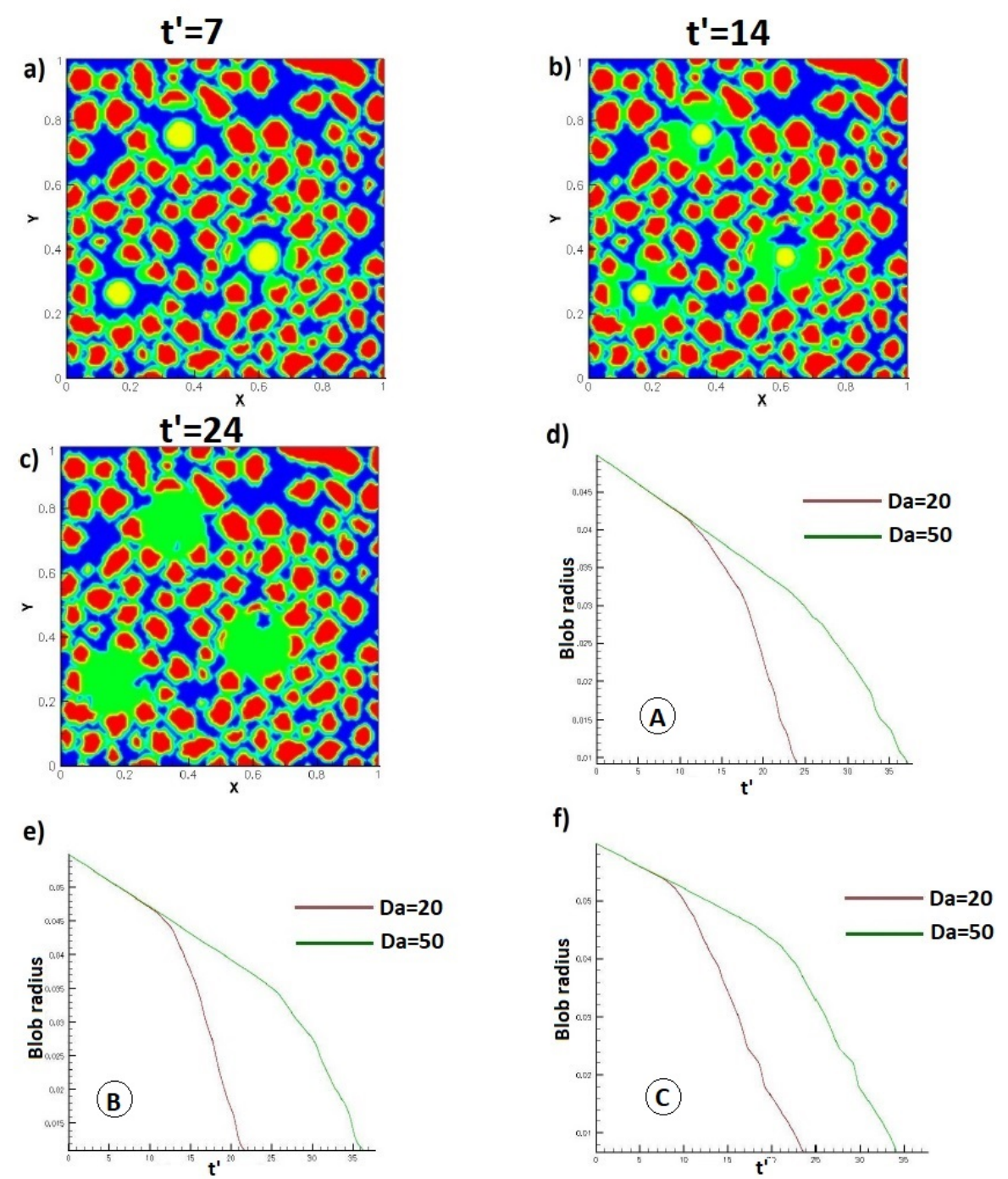

Figure 11: Evolution of the transport processes for $\left.\left.D a=50(\mathrm{a}, \mathrm{b}, \mathrm{c}): \mathrm{a}) t^{\prime}=7, \mathrm{~b}\right) t^{\prime}=14 \mathrm{c}\right) t^{\prime}=24$; temporal variation of blobs radius with two $D a$ values (d, e, f): d) blob A, e) blob B, f) blob C. 

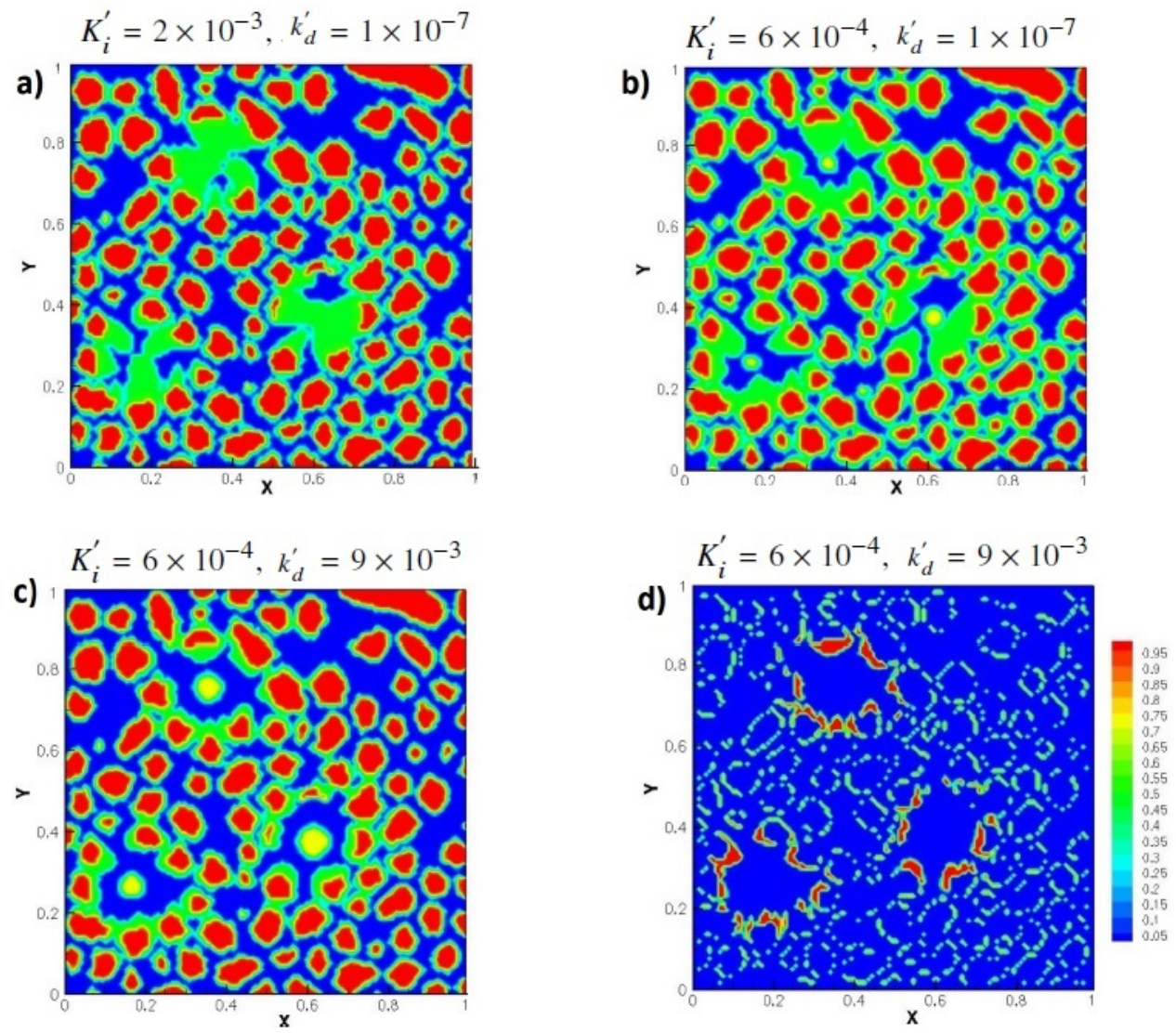

Figure 12: Evolution of the transport processes (a, b, c) : a) $\left.t^{\prime}=38, K_{i}^{\prime}=2 \times 10^{-3}, k_{d}^{\prime}=1 \times 10^{-7} \mathrm{~b}\right) t^{\prime}=38, K_{i}^{\prime}=6 \times 10^{-4}$, $k_{d}^{\prime}=1 \times 10^{-7}$ c) $t^{\prime}=38, K_{i}^{\prime}=6 \times 10^{-4}, k_{d}^{\prime}=9 \times 10^{-3}$; d) biomass concentration: $t^{\prime}=38, K_{i}^{\prime}=6 \times 10^{-4}, k_{d}^{\prime}=9 \times 10^{-3}$.

The results indicate that bacterial growth is strongly influenced by the inhibition coefficient. The more the $K_{i}$ decreases, the slower the bacterial growth moves towards the NAPL blobs. Thus, for the numerical conditions used, at $K_{i}^{\prime}=2 \times 10^{-3}$, the NAPL blobs are practically covered with bacteria and completely dissolved at $t^{\prime}=38$. On the other hand, when $K_{i}^{\prime}=6 \times 10^{-4}$, the bacteria grow preferentially far from the NAPL blobs and do not adhere to the oil phase until about $t^{\prime}=38$ (Fig. 12b).

This effect is more obvious when we increase the value of the extinction coefficient at $k_{d}^{\prime}=2 \times 10^{-4}$. The comparison performed for the same $K_{i}$ but for two different values of $k_{d}^{\prime}$ is presented in Figs. 12b and 12c. For a high value of $k_{d}^{\prime}$, bacterial growth in the vicinity of the NAPL is no longer slowed down but clearly halted, the bacterial mortality rate become higher than the growth rate above a threshold concentration of dissolved hydrocarbon. This effect can clearly be seen in Fig. 12d that indicates a decrease in the biomass 
concentration when bacteria are too far (dissolved substrate concentration too low) or too close (inhibitory effect) to the pollutant source. This creates an exclusive zone around the trapped droplets, which would become larger when $K_{i}$ is smaller and $k_{d}$ is larger.

\subsection{Upscaling of pore-scale simulations and local mass equilibrium assessment}

Numerical experiments allow us to better understand different mechanisms involved and their influence on the process of natural attenuation (favored or not by the biological activity) of a hydrocarbon source trapped within a porous medium. Another advantage of using numerical models at the pore scale is that they can validate or invalidate the relevant simplifying hypotheses in the development of macroscopic models. For example, a classical approach used in the modeling of contaminant transport is the hypothesis of local equilibrium between the fluid phase and the NAPL phase (Kaluarachchi and Parker, 1990, Ahmadi et al., 2001). This approach considers that the fluid phase surrounding the entrapped hydrocarbon droplets is close to the thermodynamic equilibrium with the NAPL phase. In other words, the dissolved hydrocarbon concentration is close to the maximum solubility at any point in the domain. Thus, solute transport in the water/NAPL system can be described by a single concentration governed by a single transport equation. This hypothesis is not always verified, since many experimental studies (Miller et al., 1990, Powers et al., 1991) have shown a non local-equilibrium behavior for which the dissolution process is remarkably longer. In this case under abiotic conditions, the hypothesis of local equilibrium between fluid and NAPL phases is irrelevant given the low saturation of NAPL. However, the suitability of the local equilibrium assumption between the fluid and biofilm phases should be assessed. It would simplify the development of the macroscopic model by switching from the three-phase system to a two-phase NAPL/water-biofilm problem. The hypothesis of local equilibrium between biofilm and fluid has been successfully validated under certain conditions in the case of dissolved contaminant transport (Golfier et al., 2009). The results of this study showed that low Péclet and Damköhler numbers are generally required to limit the occurrence of high gradients and to apply this hypothesis. However, the above work was based on an arbitrary spatial distribution of biomass which can strongly impact the occurrence of concentration gradients. We will try, in this last section, to evaluate the relevance of this hypothesis through simulations at the pore scale with our coupled model.

The geometry considered here is shown in Fig. 13a, and is constructed by duplicating four times the domain used in the previous sections. This makes the length of the domain much larger than the porescale characteristic lengths, which is enough to separate the scales and to upscale reliable correlations. The porosity of the domain and the NAPL saturation are also modified to allow a more even distribution of NAPL blobs and avoid artificially biased growth of the bacterial population. The domain now has an initial porosity $\left(\epsilon_{0}\right)$ of 0.65 and a NAPL saturation $\left(s_{0}\right)$ of $21 \%$, distributed in $(17 \times 4)$ blobs as shown in Fig. 13a.

The porous medium that we have defined above is inoculated with bacteria capable of biodegrading the hydrocarbon. The bacteria, present in sessile form, initially occupy $3.8 \%$ of the pore volume, distributed 
randomly on the solid and NAPL blob surfaces. Figure 13a shows the initial geometry of the medium inoculated by the bacteria. Biomass is illustrated here in white to facilitate readability. The simulation parameters are given in Table 4.

Table 4: Physical and biological parameters used in the simulations.

\begin{tabular}{l|l|l}
\hline Parameter & physical/biological & dimensionless value \\
& value & \\
\hline$l x$ & $48 \mathrm{~mm}$ & $l x^{\prime}=4$ \\
$l y$ & $12 \mathrm{~mm}$ & $l y^{\prime}=1$ \\
$N x$ & 465 & \\
$N y$ & 117 & \\
$c_{h}$ & $800 \mathrm{mg} / l$ & $c_{h}^{\prime}=1$ \\
$C_{e q}$ & $0.48 \mathrm{mg} / l$ & $C_{e q}^{\prime}=6 \times 10^{-4}$ \\
$D_{f}$ & $10^{-9} \mathrm{~m}^{2} / \mathrm{s}$ & $D_{f}^{\prime}=1$ \\
$D_{b}$ & $0.25 \times 10^{-9} \mathrm{~m}^{2} / \mathrm{s}$ & $D_{b}^{\prime}=0.25$ \\
$K_{f h}$ & - & $6 \times 10^{-4}$ \\
Péclet number, $(P e)$ & - & 0.3 \\
\hline$K$ & $0.24 \mathrm{mg} / l$ & $K^{\prime}=3 \times 10^{-4}$ \\
$k_{d}$ & $2 \times 10^{-14} s$ & $k_{d}^{\prime}=2 \times 10^{-7}$ \\
$K_{i}$ & $0.64 \mathrm{mg} / l$ & $K_{i}^{\prime}=8 \times 10^{-4}$ \\
$K_{b h}$ & - & $6 \times 10^{-3}$ \\
$F_{\lambda}$ & - & 0.6 \\
Damköhler number, $(D a)$ & -60 \\
\hline
\end{tabular}

Figures 13b-13c represent the results for two Damköhler numbers $D a=0.1, D a=60$ at $t^{\prime}=138$. The biofilm is rapidly developing preferentially in the vicinity of NAPL blobs leading to the formation of a biomass ring around the droplets of NAPL. At this stage, the process of dissolution is highly accelerated by increasing the solubility and bio-assimilation of the dissolved hydrocarbon. In order to determine whether the observed behavior approximates local equilibrium conditions, it must be verified that the averaged concentrations in the fluid and biofilm phases, respectively $C_{f}^{m o y}$ and $C_{b}^{\text {moy }}$, are close to equilibrium, i.e.,

$$
C_{f}^{m o y}=K_{b f} C_{b}^{m o y}
$$

We also calculate $R_{e q}=C_{f}^{\text {moy }} / K_{b f} C_{b}^{\text {moy }}$ and determine its variation compared to the NAPL saturation, as illustrated in Fig. 14.

From the beginning, for both $D a$ values, the ratio $R_{e q}$ increases around 1.1. We then observe a rapid 

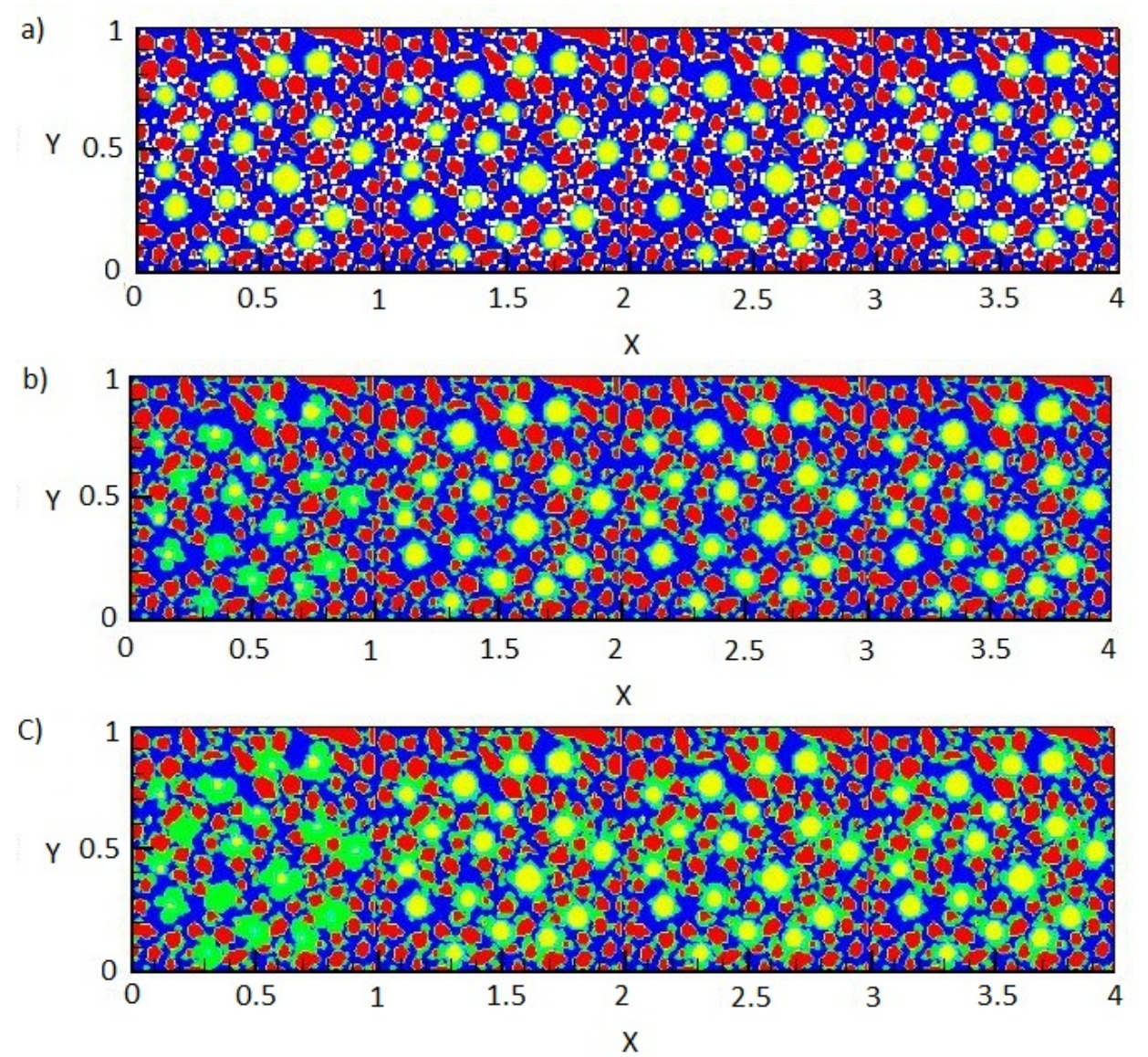

Figure 13: a) Initial geometry; b) Geometry of the porous medium for $P e=0.6$ and $D a=0.1$ at $t^{\prime}=138$; c) Geometry of the porous medium for $P e=0.6$ and $D a=60$ at $t^{\prime}=138$ characteristic behavior of non-local equilibrium, presumably linked to the preferential distribution of biofilm within the pores. Although the Péclet and Damköhler numbers are low $(P e=0.6, D a=0.1)$, the localized distribution of the bacterial droplets hinders the exchange with the fluid flow in the larger pores. The consequence of this phenomenon is a reduction of the effective interfacial surface and thus a limitation of mass transfer. These results suggest a very strong dependence of the hypothesis of local equilibrium not only to the characteristic dimensionless numbers of physical processes as classically identified but also to the pore topology. A more homogeneous distribution of biofilm related to a greater saturation of the oil phase would be necessary to approximate the local equilibrium conditions. We can also notice that, at the beginning of the simulation, the decrease of the $R_{e q}$ is almost identical for both $D a$ numbers, due to the low initial value of the volume fraction of biofilm. When the biofilm begins to develop, we observe a significant divergence between the two values of $R_{e q} . R_{e q}$ decreases to about 0.75 for $D a=0.1$, and to 0.41 for $D a=60$, which can 


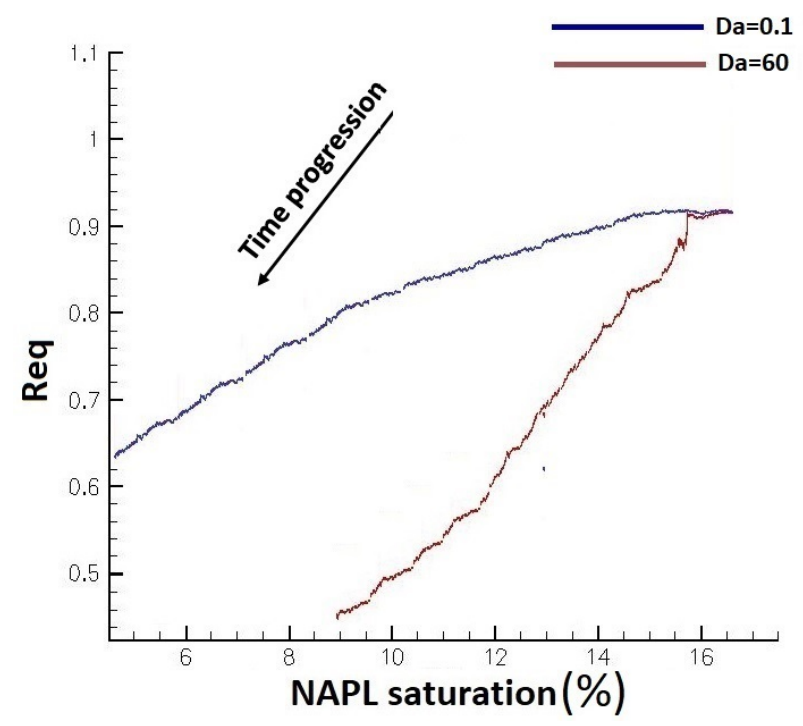

Figure 14: The evolution of $R_{e} q$ according to the NAPL saturation.

be classically explained by an increase of the non-equilibrium phenomenon for higher value of Damköhler number.

\section{Conclusions}

In this paper, we develop a 2D mathematical model integrating the mechanisms associated with the dissolution and biodegradation of NAPL phase in porous medium. Pore-scale equations of fluid flow and solute transport are combined with a biofilm growth model. Fluid flow is simulated with an immersed boundary-lattice Boltzmann model while transport of dissolved hydrocarbon is described using a Cartesian cut-cell method. A cellular automaton algorithm based on the immersed boundary method previously developed in (Benioug et al., 2017) is used to describe the spreading and distribution of biomass. A uniform dissolution approach, which we consider as the most suitable for the envisaged simulations, is adopted to describe the evolution of the residual NAPL droplets over time.

The first set simulations focus on NAPL dissolution in abiotic conditions with analysis under different hypotheses (droplets size and Péclet number) and the effect of the spatial distribution of NAPL droplets on the dissolution rate. The results obtained are consistent with those from the literature. In a second step, we are interested in the bioenhancement of NAPL dissolution. Different conditions are also considered (spatial distribution, reaction kinetics, toxicity), and their impacts on the process of dissolution in the presence of bacteria are investigated. Finally, this pore-scale model is used to assess the suitability of a local equilibrium assumption between the fluid-biofilm phases in the vicinity of the NAPL sources. Despite the 
scarce numerical or experimental results available, our model is in agreement with the description of the physical phenomena expected and studied through the examples considered.

\section{Acknowledgements}

M. Benioug and X. Yang are funded by the Recruitment Program of Global Experts in China. This work was partially supported by the French National Research Agency (ANR) through the MOBIOPOR project, with the reference ANR-10-BLAN-0908 and was performed as part of the French Scientific Interest Group-Industrial Wasteland (GISFI) program. This project has also received partial funding from the European Union's Horizon 2020 research and innovation program through the PROTINUS project under Grant Agreement No. 645717. 


\section{Reference}

Ahmadi, A., A. Aigueperse, and M. Quintard (2001), Calculation of the effective properties describing active dispersion in porous media: from simple to complex unit cells, Advances in Water Resources, 24, 423-438.

Al-Bader, D., M. Kansour, R. Rayan, and S. Radwan (2013), Biofilm comprising phototrophic, diazotrophic, and hydrocarbonutilizing bacteria: a promising consortium in the bioremediation of aquatic hydrocarbon pollutants, Environmental Science and Pollution Research, 20(5), 3252-3262.

Armstrong, R. T., and D. Wildenschild (2012), Investigating the pore-scale mechanisms of microbial enhanced oil recovery, Journal of Petroleum Science and Engineering, 94, 155-164.

Bahar, T., F. Golfier, C. Oltéan and M. Benioug (2016), An upscaled model for bio-enhanced NAPL dissolution in porous media, Transport in Porous Media, 113(3), 653-693.

Benioug, M., F. Golfier, A.-J. Tinet, M. A. Buès, and C. Oltéan (2015), Numerical efficiency assessment of IB-LB method for 3D pore-scale modeling of flow and transport, Transport in Porous Media, 109(1), 1-23.

Benioug, M., F. Golfier, C. Oltéan, M. Buès, T. Bahar, and J. Cuny (2017), An immersed boundary-lattice Boltzmann model for biofilm growth in porous media, Advances in Water Resources, 10\%, 65 - 82 .

Brackman, G., U. Hillaert, S. Van Calenbergh, H. Nelis, and T. Coenye (2009), Use of quorum sensing inhibitors to interfere with biofilm formation and development in Burkholderia multivorans and Burkholderia cenocepacia, Research in Microbiology, $160(2), 144-151$.

Chmielewski, R., and J. Frank (2003), Biofilm formation and control in food processing facilities, Comprehensive Reviews in Food Science and Food Safety, 2(1), 22-32.

Chu, M., P. Kitanidis, and P. McCarty (2004), Possible factors controlling the effectiveness of bioenhanced dissolution of non-aqueous phase tetrachloroethene, Advances in Water Ressources, 27, 601-615.

Decho, A. (2000), Microbial biofilms in intertidal systems: an overview, Continental Shelf Research, $20(10$ - 11$), 1257$ - 1273.

De Rosis, Alessandro (2014), Harmonic oscillations of laminae in non-Newtonian fluids: A lattice Boltzmann-Immersed Boundary approach, Advances in Water Resources, 73, 97-107.

Eberl, H., C. Picioreanu, J. Heijnen, and M. van Loosdrecht (2000), A three-dimensional numerical study on the correlation of spatial structure, hydrodynamic conditions, and mass transfer and conversion in biofilms, Chemical Engineering Science, $55(24), 6209-6222$.

Eberl, H., D. Parker, and M. van Loosdrecht (2001), A new deterministic spatio-temporal continuum model for biofilm development, Journal of Theoretical Medicine, 3, 161-175.

Ebigbo, A., F. Golfier, and M. Quintard (2013), A coupled pore-scale model for methanogenic microbial activity in underground hydrogen storage, Advances in Water Resources, 61, 74-85.

Gallo, C. and S. M. Hassanizadeh (2002), Modeling NAPL dissolution and biodegradation interactions: effect of toxicity and biomass growth limitations, Developments in Water Science, 47, 859-866.

Golfier, F., B. Wood, L. Orgogozo, M. Quintard, and M. Buès (2009), Biofilms in porous media: Development of macroscopic transport equations via volume averaging with closure for local mass equilibrium conditions, Advances in Water Resources, 32(3), 463-485.

Huang, T., J. Xu, and D. Cai (2011), Efficiency of active barriers attaching biofilm as sediment capping to eliminate the internal nitrogen in eutrophic lake and canal, Journal of Environmental Sciences, 23(5), 738 - 743.

Imhoff, P. T., P. R. Jaffé, and G. F. Pinder (1994), An experimental study of complete dissolution of a nonaqueous phase liquid in saturated porous media, Water Resources Research, 30, 307-320.

Ingram, D. M., D. M. Causon, and C. G. Mingham (2003), Developments in Cartesian cut cell methods, Mathematics and Computers in Simulation, 61(3-6), 561-572. 
Kaluarachchi, J., and J. Parker (1990), Modeling multi-component organic chemical transport in three-fluid-phase porous media, Journal of Contaminant Hydrology, 5, 349-374.

Khosronejad, A., Kang, S. Borazjani, I and Sotiropoulos, F (2011), Curvilinear immersed boundary method for simulating coupled flow and bed morphodynamic interactions due to sediment transport phenomena, Advances in Water Resources, $34(7), 829-843$

Kennedy, C. A., and W. C. Lennox (1997), A pore-scale investigation of mass transport from dissolving DNAPL droplets, Journal of Contaminant Hydrology, 24, 221-246.

Kent, B., and M. Bianchi (2001), Remediation of NAPL-contaminated aquifers: is the cure worth the cost?, Journal of Environmental Science and Health, 36, 1559-1569.

Kreft, J.-U., G. Booth, and J. Wimpenny (1998), BacSim, a simulator for individual-based modelling of bacterial colony growth, Microbiology, 144, 3275-3287.

Krefta, J.-U., C. Picioreanu, J. Wimpenny, and M. van Loosdrecht (2001), Individual-based modelling of biofilms, Microbiology, $147,2897-2912$.

Kumar, A., S. Kumar, and S. Kumar (2005), Biodegradation kinetics of phenol and catechol using Pseudomonas putida MTCC 1194, Biochemical Engineering Journal, 22, 151-159.

Lee, Y.-C., T.-S. Kwon, J.-S. Yang, and J.-W. Yang (2007), Remediation of groundwater contaminated with DNAPLs by biodegradable oil emulsion, Journal of Hazardous Materials, 140, 340-345.

Mah, T., and G. O'Toole (2001), Mechanisms of biofilm resistance to antimicrobial agents, Trends in Microbiology, 9, 39-41.

Marschall, H., K. Hinterberger, C. Schüler, F. Habla, and O. Hinrichsen (2012), Numerical simulation ofspecies transfer across fluid interfaces in free-surface flows using OpenFOAM, Chemical Engineering Science, 78, 111-127.

Mateas, D. J., G. R. Tick, and K. C. Carroll (2017), In situ stabilization of NAPL contaminant source-zones as a remediation technique to reduce mass discharge and flux to groundwater, Journal of Contaminant Hydrology, 204(Supplement C), 40 56.

Mercer, J., and R. Cohen (1990), A review of immiscible fluids in the subsurface: Properties, models, characterization and remediation, Journal of Contaminant Hydrology, 6, 107-163.

Miller, C., M. Poirier-McNeill, and A. Mayer (1990), Dissolution of trapped nonaqueous phase liquids: Mass transfer characteristics, Water Resources Research, 26, 2783-2796.

Miller, C., G. Christakos, P. Imhoff, J. McBride, J. Pedit, and J. Trangenstein (1998), Multiphase flow and transport modeling in heterogeneous porous media: challenges and approaches, Advances in Water Resources, 21, 77-120.

Mohd-Yusof J (1997), Combined immersed boundaries/B-spline methods for simulations of flows in complex geometries, CTR Annual Research Briefs, NASA Ames/Stanford University, 317-327.

Paulsen, J. E., S. Ekrann, and E. Oppen (1989), Visualisation of bacterial degradation and mobilisation of oil in a porous medium, Environmental Geology, 38, 204-208.

Picioreanu, C., M. van Loosdrecht, and J. Heijnen (2000a), A theoretical study on the effect of surface roughness on mass transport and transformation in biofilms., Biotechnology Bioengineering, 68, 355-369.

Picioreanu, C., M. van Loosdrecht, and J. Heijnen (2000b), Effect of diffusive and convective substrate transport on biofilm structure formation: a two-dimensional modeling study, Biotechnology Bioengineering, 69, 504-515.

Poesio, Pietro, Gian Paolo Beretta, and Todd Thorsen (2009), Dissolution of a Liquid Microdroplet in a Nonideal Liquid-Liquid Mixture Far from Thermodynamic Equilibrium, Physical Review Letters, 103(6), 064501.

Powers, S., C. Loureiro, L. Abriola, and W. Weber (1991), Theoretical study of the significance of nonequilibrium dissolution of nonaqueous phase liquids in subsurface systems, Water Resources Research, 27, 463-477.

Powers, S., L. Abriola, and W. Weber (1992), An experimental investigation of nonaqueous phase liquid dissolution in saturated subsurface systems: Steady state mass transfer rates, Water Resources Research, 28, 2691-2705. 
Powers, S., L. Abriola, and W. Weber (1994), An experimental investigation of nonaqueous phase liquid dissolution in saturated subsurface systems: Transient mass transfer rates, Water Resources Research, 30, 321-332.

Saravanan, P., K. Pakshirajan, and P. Saha (2008), Growth kinetics of an indigenous mixed microbial consortium during phenol degradation in a batch reactor, Bioresource Technology, 99, 205-209.

Seagren, E.A., B.E. Rittmann and A.J. Valocchi (1994), Quantitative evaluation of the enhancement of NAPL-pool dissolution by flushing and biodegradation, Environmental Science \& Technology, 28(5), 833-839.

Seo, Y., W.-H. Lee, G. Sorial, and P. Bishop (2009), The application of a mulch biofilm barrier for surfactant enhanced polycyclic aromatic hydrocarbon bioremediation, Environmental Pollution, 157(1), 95 - 101.

Singh, R., and M. Olson (2010), Kinetics of trichloroethylene and toluene toxicity to Pseudomonas putida F1., Environmental Toxicology and Chemistry, 29, 56-63.

Singh, R., D. Paul, and R. Jain (2006), Biofilms: implications in bioremediation, Trends in Microbiology, 14,389 - 397.

Succi, S. (2011), The lattice Boltzmann equation for fluid dynamics and beyond, Oxford : Clarendon Press.

van Loosdrecht, M., J. Heijnen, H. Eberl, J.-U. Kreft, and C. Picioreanu (2002), Mathematical modelling of biofilm structures, Antonie van Leeuwenhoek, 81 (1-4), 245-256.

Vega, L., J. Mathieu, Y. Yang, B. Pyle, R. McLean, and P. Alvarez (2014), Nickel and cadmium ions inhibit quorum sensing and biofilm formation without affecting viability in Burkholderia multivorans, International Biodeterioration EG Biodegradation, $91(0), 82-87$.

Wingender, J., T. Neu, and H.-C. Flemming (1999), What are bacterial extracellular polymeric substances?, in Microbial Extracellular Polymeric Substances, edited by J. Wingender, T. Neu, and H.-C. Flemming, pp. 1-19, Springer Berlin Heidelberg.

Yang, Y., and P. L. McCarty (2000), Biologically enhanced dissolution of tetrachloroethene DNAPL, Environmental Engineering and Science, 34(14), 2979-2984. 